\title{
Fundamental in vitro 3D human skin equivalent tool development for assessing biological safety and biocompatibility - towards alternative for animal experiments
}

\author{
Ayesha Idrees ${ }^{1,2,3}$, Inge Schmitz ${ }^{4}$, Alice Zoso ${ }^{1,5}$, Dierk Gruhn ${ }^{3}$, Sandra Pacharra ${ }^{3}$, Siegfried Shah ${ }^{3}$, \\ Gianluca Ciardelli ${ }^{1}$, Richard Viebahn ${ }^{2,3}$, Valeria Chiono ${ }^{1,5, *, * *}$, and Jochen Salber ${ }^{2,3, *, * *}$ \\ ${ }^{1}$ Department of Mechanical and Aerospace Engineering (DIMEAS), Politecnico di Torino, 10129 Turin, Italy \\ ${ }^{2}$ Universitätsklinikum Knappschaftskrankenhaus Bochum, Department of Surgery, Hospital of the RUHR-University, \\ 44892 Bochum, Germany \\ ${ }^{3}$ Department of Experimental Surgery, Centre for Clinical Research, RUHR-University, 44801 Bochum, Germany \\ ${ }^{4}$ Institute of Pathology, RUHR University, 44789 Bochum, Germany \\ ${ }^{5}$ Interuniversity Centre for the Promotion of the 3Rs Principles in Teaching and Research, Centro 3R, 56122 Pisa, Italy
}

Received 17 December 2020, Accepted 3 February 2021

\begin{abstract}
Nowadays, human skin constructs (HSCs) are required for biomaterials, pharmaceuticals and cosmetics in vitro testing and for the development of complex skin wound therapeutics. In vitro threedimensional (3D) dermal-epidermal based interfollicular, full-thickness, human skin equivalent (HSE) was here developed, recapitulating skin morphogenesis, epidermal differentiation, ultra-structure, tissue architecture, and barrier function properties of human skin. Different 3D cell culture conditions were tested to optimize HSE maturation, using various commercially available serum/animal component-free and/or fully defined media, and air-liquid interface (ALI) culture. Optimized culture conditions allowed the production of HSE by culturing normal human dermal fibroblasts (NHDFs) for 5-7 days in CELLnTEC-Prime Fibroblast (CnT-PR-F) medium and then culturing normal human epidermal keratinocytes (NHEKs) for 3 days in CELLnTEC-Prime Epithelial culture (CnT-PR) medium on them. Co-culture was then submerged overnight in CELLnTEC-Prime-3D barrier (CnT-PR-3D) medium to stimulate cell-cell contact formation and finally placed at ALI for 15-20 days using CnT-PR-3D medium. Histological analysis revealed uniform distribution of NHDFs in the dermal layer and their typical elongated morphology with filopodia. Epidermal compartment showed a multi-layered structure, consisting of stratum basale, spinosum, granulosum, and corneum. NHDFs and keratinocytes of basal layer were positive for the proliferation marker Kiel 67 (Ki-67) demonstrating their active state of proliferation. The presence of typical epidermal tissue proteins (keratins, laminins, filaggrin, loricin, involucrin, and $\beta$-tubulin) at their correct anatomical position was verified by immunohistochemistry (IHC). Moreover, transmission electron microscopy (TEM) analyses revealed basement membrane with lamina lucida, lamina densa, hemidesmosomes and anchoring fibers. The epidermal layers showed abundant intracellular keratin filaments, desmosomes, and tight junction between keratinocytes. Scanning electron microscopy (SEM) analyses showed the interwoven network of collagen fibers with embedded NHDFs and adjacent stratified epidermis up to the stratum corneum similar to native human skin. HSE physiological static contact angle confirmed the barrier function. The developed HSE represents a fundamental in vitro tool to assess biocompatibility of biomaterials, pharmacotoxicity, safety and effectiveness of cosmetics, as well as to investigate skin biology, skin disease pathogenesis, wound healing, and skin infection.
\end{abstract}

Keywords: 3D, Actin, Animal model, Biocompatibility, Biomaterials, Collagen, Culture, Dermal, Dermatoblasts, Development, ECM, Electron microscopy, Extracellular matrix, Effectiveness, Engineering, Epidermal, Equivalent, Fibroblasts, Flg, Human skin equivalent, HSE, Inv, iPSC, Keratinocytes, KGF, Lam, Layer, Lor, Maturation, Medium, Microenvironment, Organoid, NHDF, NHEK, Pharmacotoxicity, Proliferation, Regenerative medicine, SE, Signaling, TEM, Tissue engineering, Wound healing

\footnotetext{
* Corresponding authors: valeria.chiono@polito.it; jochen.salber@rub.de

** These authors have contributed equally to this work and share senior authorship.
} 


\section{Introduction}

Human skin constructs (HSCs) are three-dimensional (3D) in vitro tissue-engineered human skin, which may find application in regenerative medicine and as in vitro models for fundamental research and industrial uses, for example for cytotoxicity analysis, and testing new therapeutic approaches. In advanced $3 \mathrm{D}$ in vitro systems, keratinocytes are able to develop well-ordered epidermis and basement membrane features [1-3], to closely recapitulate the characteristics of native human skin (NHS). On the other side, fibroblasts have an important role in enhancing the keratinocytes resistance towards toxic compounds [4]. This suggests that a single compartment skin model may not be predictive for in vitro toxicological studies.

As HSCs are physiologically more similar to the NHS, they offer advanced 3D testing systems as an alternative to animals for drugs and cosmetics evaluation, irritancy and toxicity testing, wound healing studies, cancer research, skin infection studies, and research on other skin diseases [5-7]. Human and animal skin (e.g. mouse) have different structures, functionalities and responsiveness. Murine epidermis is quite thin composed of only three layers with a high turnover rate and present a cutaneous muscle layer, while human epidermis is thick, composed of six to ten layers but lacks the muscle layer [8]. Moreover, mouse skin is effectively able to regenerate after wounding, while human skin damage may lead to hypertrophic scar (keloids) formation [9].

Replacing low- to non-transferable in vivo animal experiments and in vitro $3 \mathrm{D}$ animal skin models with in vitro $3 \mathrm{D}$ skin models constructed from human cells is in line with the European Union regulations, 7th amendment (Dir. 2003/15/EC) of the "Cosmetics Directive" (76/768/ EEC) which made obligatory to replace animal trials for cutaneous resorption with reliable in vitro tests by the year 2009 [10], leading to the development of the 3Rs principle "Replacement, Reduction and Refinement" [11].

Extracellular matrix (ECM) is the mechanical support and main component of 3D tissue microenvironment. The ECM also serves as a lead structure for diffusible molecules, including growth factors, which bind to ECM macromolecules and regulate inter- and intracellular communication via signalling pathways. It plays a key role in physiological tissue homeostasis, growth and differentiation, but in pathological processes as well. Furthermore, the mutual interactions between ECM and cells by mechanotransduction, result in specific cell surface receptor expression (e.g. integrins) [12], metabolic functions, cell proliferative potential, ECM production and release of key regulators [13-20]. 2D cell cultures fail to reproduce these features and result in non-predictive outcomes mainly due to forcing cells to adapt to an artificial flat 2D surface [21]. Cells grown in 3D environment may replicate the relevant complexity and dynamicity of the in vivo microenvironment.

Up to now, studies on in vitro engineered human dermal-epidermal based skin models have been limited, while simple but poorly predictive epidermal models have been widely investigated [22]. Although progresses have been made on differentiating mouse and human induced pluripotent stem cells (iPSCs) in fully functional skin cells [23], and human skin organoids have been developed [24], these models completely submerged in medium are not able to recapitulate the physiological air-liquid interface environment or may still contain a heterogenous cell population with undifferentiated stem cells [24, 25].

Advances in tissue engineering, cell transplantation, and gene therapy allow to obtain pure human cell cultures with high quality. However, the standardization of the procedures, especially for proliferative keratinocytes preparation as well as for their maintenance is a major obstacle. Additionally, the use of serum and other medium components is associated with batch-to-batch variations [26]. Animalfree compounds and recombinant human growth factors could represent a valuable alternative [27].

In this study, 3D cell culture conditions (3D-CCs) and culture at air-liquid interface (ALI) were optimized to develop full-thickness human skin equivalent (HSE) using human primary cells. To achieve this aim, a gradual shift towards the use of serum- and animal component-free cell culture media was performed to optimize HSE development to obtain thus dermal-epidermal constructs closely mimicking human skin.

The HSE was based on a dermal compartment consisting of fibroblasts embedded in collagen type I (Col. I) and a multi-layered, well differentiated epidermal compartment. Unlike epidermal or dermal skin models, full-thickness skin models comprising both dermal and epidermal compartments are advantageous. Firstly, fibroblasts secrete growth factors (e.g. keratinocyte growth factor, KGF) which directly influence the growth and differentiation of keratinocytes [3, 28, 29]. Soluble factors secreted by dermal fibroblasts are diffused to the overlying epidermis, influencing the keratinocytes to induce the synthesis of basement membrane proteins (i.e. laminin, collagen type IV, perlecan and nidogen) [1, 3, 30-32]. On the other hand, keratinocytes by secreting interleukin-1 (IL-1), also directly influence the proliferation of fibroblasts [29]. Moreover, the interaction between these two cell types is also highly important in the formation of the basement membrane as a part of dermal-epidermal junction (DEJ) [33, 34]. Thus, the reciprocal interaction works as a double-paracrine mechanism regulating each cell type $[35,36]$. This cell-cell communication is mediated by hormones, growth factors, cytokines and other signalling molecules that are secreted by a cell and act on cells in its immediate vicinity and vice versa.

The goal of this work was to develop an open-source and well-reproducible 3D HSC as in vitro model of human full skin, that can be helpful for preclinical testing, in compliance with the 3 Rs principle.

\section{Materials and methods \\ Cell source and maintenance}

Primary cells including normal human dermal fibroblasts (NHDF; CD90 positive) and normal human epidermal keratinocytes (NHEK; Cytokeratin positive) were obtained 
from PromoCell. The company guaranteed the following requested specifications: Same batch, single adult donor, chest region, and passage number $\mathrm{P} 2$ after thawing.

NHDF and NHEK were maintained in CnT-Prime fibroblast medium (CnT-PR-F, CELLnTECH) and CnTPrime epithelial culture medium (CnT-PR, CELLnTECH) respectively under the physiological culture conditions $\left(37^{\circ} \mathrm{C}, 5 \% \mathrm{CO}_{2}\right)$, and sub-cultured using DetachKit-PromoCell HEPES BSS (2-[4-(2-hydroxyethyl)piperazine-1-yl] ethanesulfonic acid buffered saline solution); $0.04 \%$ Tryp$\sin / 0.03 \%$ EDTA (ethylenediaminetetraacetic acid); and TNS (Trypsin Neutralizing Solution) containing 0.05\% trypsin inhibitor from soybean $/ 0.1 \%$ bovine serum albumin. Collagen type I (Col. I) from rat tail tendons, $5 \mathrm{mg} / \mathrm{mL}$ stock concentration was purchased from Ibidi.

Different media were used to optimize the development of HSE: i) CnT-PR-F (CnT-Prime fibroblast medium, CELLnTECH) named as Medium A; ii) CnT-PR (CnTPrime epithelial culture medium, CELLnTECH) named as Medium B; iii) CnT-PR-3D (CnT-Prime-3D barrier medium, CELLnTECH) named as Medium C; iv) CnTPR-FTAL (CnT-Prime-full thickness airlift medium, CELLnTECH); v) KGM2 (Keratinocyte Growth Medium 2, i.e. Basal medium supplemented with Bovine Pituitary Extract $0.004 \mathrm{~mL} / \mathrm{mL}$, human EGF $0.015 \mathrm{ng} / \mathrm{mL}$, human Insulin $5 \mu \mathrm{g} / \mathrm{mL}$, Hydrocortisone $0.33 \mu \mathrm{g} / \mathrm{mL}$, Epinephrine $0.39 \mu \mathrm{g} / \mathrm{mL}$, Transferrin $10 \mu \mathrm{g} / \mathrm{mL}, \mathrm{CaCl}_{2} 0.06 \mathrm{mM}$; PromoCell); vi) High calcium KGM2 (KGM2 + $1.2 \mathrm{mM} \mathrm{CaCl}_{2}$, Sigma); vii) FGM2 is a low serum media $(2 \% \mathrm{v} / \mathrm{v})$ that contains: $0.02 \mathrm{~mL} / \mathrm{mL}$ foetal calf serum (FCS), $1 \mathrm{ng} / \mathrm{mL}$ basic fibroblasts growth factor (bFGF) (recombinant human), $5 \mu \mathrm{g} / \mathrm{mL}$ Insulin (recombinant human) (PromoCell).

\section{Preparation of Human Skin Equivalents (HSEs)}

The 3D HSEs were generated by successively fabricating dermal and epidermal layers as summarized in Figure 1A.

\section{Preparation of Human Dermal Constructs (HDCs)}

Gelation of Col. I solution was performed in $10 \times$ media (M199, Sigma) supplemented with L-glutamine and sodium bicarbonate $\left(\mathrm{NaHCO}_{3}\right)$, resulting in a final Col. I concentration of $1.5 \mathrm{mg} / \mathrm{mL}$ (containing a salt concentration of $1 \times$ in final mixture with $\mathrm{pH}$ of 7.2-7.4) (Tab. 1)

HDCs were prepared by fabricating acellular $(200 \mu \mathrm{L})$ and cell containing layers $(400 \mu \mathrm{L})$ of Col. I matrix on polyester membrane of 12 well inserts (Corning 3460, $0.4 \mu \mathrm{m}$ pore size, $12 \mathrm{~mm}$ diameter, $1.12 \mathrm{~cm}^{2}$ surface area) to obtain $5 \mathrm{~mm}$ thick HDCs. A thin layer of acellular collagen served as a substrate for the cellular collagen, preventing the cellular collagen from complete contraction from the insert membrane. Actively dividing mitotic cells $\left(5 \times 10^{4} \mathrm{NHDF}\right.$ cells per $12 \mathrm{~mm}$ diameter insert to obtain the human in vitro dermal construct) were embedded in Col. I solution and added on the top of the previously deposited and gelled acellular Col. I layer (room temperature, $20 \mathrm{~min}$ ). After gelation of cellularized matrix $\left(37^{\circ} \mathrm{C}\right.$ for $\left.30 \mathrm{~min}\right)$, fresh cell culture medium (Medium A) was added and incubated for 5-7 days to allow hydrogel remodelling by the embedded cells (Figs. 2A and 2B).

\section{Development of epidermis onto human dermal constructs}

Media was removed from HDC 20 min before NHEK seeding. NHEK were resuspended (400,000 cells for a surface area of dermal construct of $12 \mathrm{~mm}$ diameter) in a small volume of $50 \mu \mathrm{L}$ KGM2 (3D-CC-I and II), CnT-PR (3DCC-III) or CnT-PR-FTAL (3D-CC-IV) in the center and plated onto the matrix for cultures epithelialization. Plates were not moved for $15 \mathrm{~min}$ to allow keratinocytes to initiate adhesion and then placed at $37{ }^{\circ} \mathrm{C}$ for $30-60$ min without medium for complete adhesion. Depending on the selected culture conditions 3D-CC-I-IV, the plates were fed with the appropriate media KGM2, CnT-PR (Medium B) or CnT-PR-FTAL as shown in Table 2. The Transwell inserts were placed in 12 well cell culture plate (Corning 3513), thus providing medium volumes of $\sim 0.3 \mathrm{~mL}$ inside and of $\sim 1.7 \mathrm{~mL}$ outside the insert. Medium was changed daily by discarding first medium from outside the insert and then from inside; fresh medium was then added initially inside the insert and then outside. Cultures were maintained in submerged conditions for 3-4 days to allow complete coverage to form a monolayer. In case of culture condition 3DCC-II KGM2 was replaced with CnT-PR-3D (Medium C) and for 3D-CC-III Medium B was replaced with Medium $\mathrm{C}$ (Tab. 2). For both culture conditions cultures were kept submerged overnight (15-16 h) to allow cells to form intercellular adhesion structures. Finally, the medium was aspirated from inside the insert to allow ALI culture for 20 days (Figs. 2C-2E). During ALI, medium was changed 3 times/ week using a minimum volume of $1 \mathrm{~mL}$.

Different 3D cell culture conditions (namely 3D-CC-I, -II, -III, -IV) based on different types of commercially available media were tested to optimize HSE development. The details of the 3D-CC are reported in Table 2. The test setup is described as timeline in Figure 1B.

In this study, the same batch (single donor, chest region) and passage number ( $\mathrm{P} 2$ after thawing) of fibroblasts and keratinocytes were used for optimizing the HSE development under different 3D culture conditions, thus eliminating the variability associated with batch-to-batch variation.

\section{Morphological analysis}

Harvested HSE tissue was rinsed in PBS twice, fixed in $4 \%$ buffered paraformaldehyde (PFA, $\mathrm{pH} 6.8-7.0$ ) for $\geq$ $4 \mathrm{~h}$, dehydrated through increasing gradient series of ethanol $(50 \%, 70 \%, 80 \%, 90 \%, 95 \%, 100 \%, 100 \%, 100 \%$; each for $5 \mathrm{~min}$ ), cleared in xylol (three times, each for $5 \mathrm{~min}$ ), and infiltrated in paraffin using Leica TP1020 Semi-enclosed Benchtop Tissue Processor (over a total period of $12 \mathrm{~h}$ ). The processed tissue was paraffin embedded at right orientation using Leica EG1160 Embedding Centre. The sample was thinly sliced $(\sim 5 \mu \mathrm{m})$ using Leica RM 2155 microtome. Sections were dryed at $37{ }^{\circ} \mathrm{C}$ overnight. Morphological 


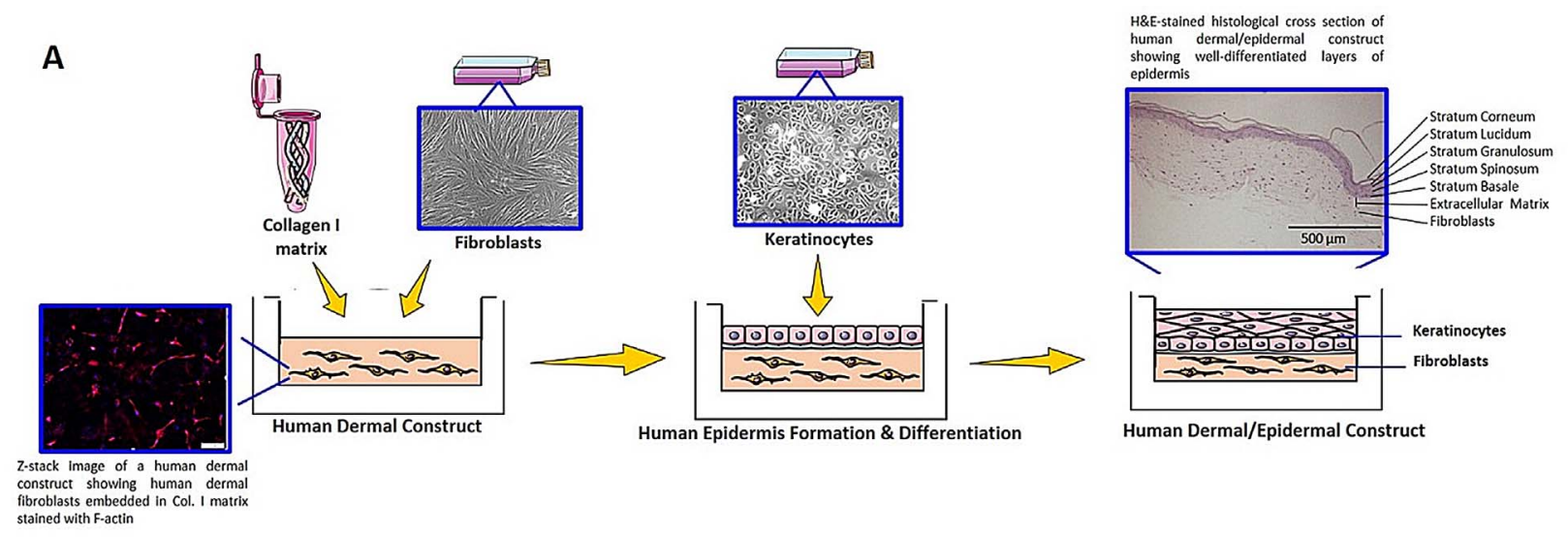

B

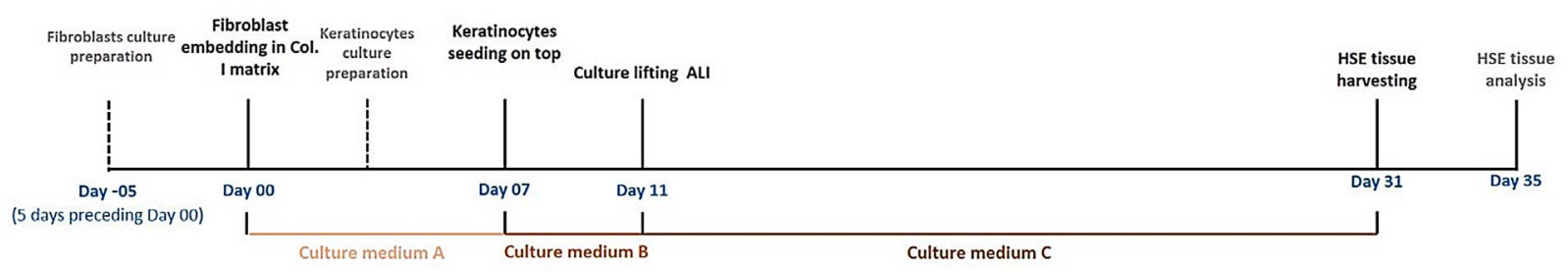

Three-dimensional culture condition-III (3D-CC-III)

Fig. 1. (A) Schematic overview of methodology for the development of HSE. It starts with the embedding of human dermal fibroblasts in collagen I matrix to contract and remodel the gel to form dermal layer. Then the keratinocytes are seeded on the top to form a monolayer and the culture is raised to ALI under 3D cell culture conditions allowing the keratinocytes to differentiate and form epidermal layers. (B) Timeline for the development of HSE.

Table 1. Guidelines for construction of collagen I $(1.5 \mathrm{mg} / \mathrm{mL})$ based dermal layer.

\begin{tabular}{|c|c|c|}
\hline \multirow[t]{2}{*}{ Constituents } & Calculated vol. of each constituent $(\mu \mathrm{L})$ & Calculated vol. of each constituent $(\mu \mathrm{L})$ \\
\hline & Total vol. of acellular layer $=200 \mu \mathrm{L}$ per insert & Total vol. of cellular layer $=400 \mu \mathrm{L}$ per insert \\
\hline 10× M199 (Sigma) & $\begin{array}{l}20 \mu \mathrm{L} \\
(10 \% \text { of final vol. of } 200 \mu \mathrm{L} \text { results in } 1 \times \mathrm{M} 199)\end{array}$ & $\begin{array}{l}40 \mu \mathrm{L} \\
(10 \% \text { of final vol. of } 400 \mu \mathrm{L} \text { results in } 1 \times \mathrm{M} 199)\end{array}$ \\
\hline $200 \mathrm{mM}$ Glutamine & $0.68 \mu \mathrm{L}$ & $1.36 \mu \mathrm{L}$ \\
\hline$(\operatorname{Bios}$ & $\begin{array}{l}\text { Formula: } \mathrm{M} 1 \cdot \mathrm{V} 1=\mathrm{M} 2 \cdot \mathrm{V} 2(200 \mathrm{mM} \times \\
\mathrm{V} 1=0.68 \times 200 \mu \mathrm{L})(\mathrm{This} 0.68 \mu \mathrm{L} \text { vol. results in } 0.68 \\
\mathrm{mM} \text { Glutamine in final } \\
200 \mu \mathrm{L} \text { vol. of acellular layer })\end{array}$ & $\begin{array}{l}\text { Formula: } \mathrm{M} 1 \cdot \mathrm{V} 1=\mathrm{M} 2 \cdot \mathrm{V} 2(200 \mathrm{mM} \times \mathrm{V} 1=0.68 \times \\
400 \mu \mathrm{L})(\mathrm{This} 1.36 \mu \mathrm{L} \text { vol. results in } 0.68 \mathrm{mM} \text { Glutamine } \\
\text { in final } 400 \mu \mathrm{L} \text { vol. of cellular layer })\end{array}$ \\
\hline $\mathrm{dd}_{2} \mathrm{O}$ & $\begin{array}{l}113.32 \mu \mathrm{L} \\
\text { (vol. calculated in the end) }\end{array}$ & $\begin{array}{l}176.64 \mu \mathrm{L} \\
\text { (vol. calculated in the end) }\end{array}$ \\
\hline $\begin{array}{l}7.5 \% \text { of } \mathrm{NaHCO}_{3} \\
\text { (Sigma) }\end{array}$ & $\begin{array}{l}6 \mu \mathrm{L} \\
\text { (This volume is } 10 \% \text { of the volume of collagen I, that } \\
\text { results in a final mixture } \mathrm{pH} \text { of } 7.2-7.4 \text { - optimized) }\end{array}$ & $\begin{array}{l}12 \mu \mathrm{L} \\
\text { (This is an optimized volume as } 10 \% \text { of the volume of } \\
\text { collagen I, that results in a final mixture } \mathrm{pH} \text { of } 7.2-7.4 \text { ) }\end{array}$ \\
\hline $\begin{array}{l}5 \mathrm{mg} / \mathrm{mL} \text { Collagen I } \\
\text { (Ibidi) }\end{array}$ & $\begin{array}{l}60 \mu \mathrm{L} \\
\text { (This volume makes final conc. } \\
\text { of } 1.5 \mathrm{mg} / \mathrm{mL} \text { in final volume) }\end{array}$ & $\begin{array}{l}120 \mu \mathrm{L} \\
\text { (This volume makes final conc. of } 1.5 \mathrm{mg} / \mathrm{mL} \text { in final } \\
\text { volume) }\end{array}$ \\
\hline Total vol. & $200 \mu \mathrm{L}$ & $400 \mu \mathrm{L}$ \\
\hline
\end{tabular}

analysis was performed on formalin-fixed paraffinembedded sections through Haematoxylin and Eosin (H\&E) staining following a standard protocol [37]. The H\&E stained slides were examined with Olympus BX51 light microscope. NHDF embedded in Col. I (Fig. 1A) were visualized by applying F-actin/nuclei staining. This was performed using Phalloidin/DAPI stain from Promokine following a standard protocol [47]. The fluorescence microscopy and Z-stack imaging was performed using Olympus XM10 with cellSense Standard software. 


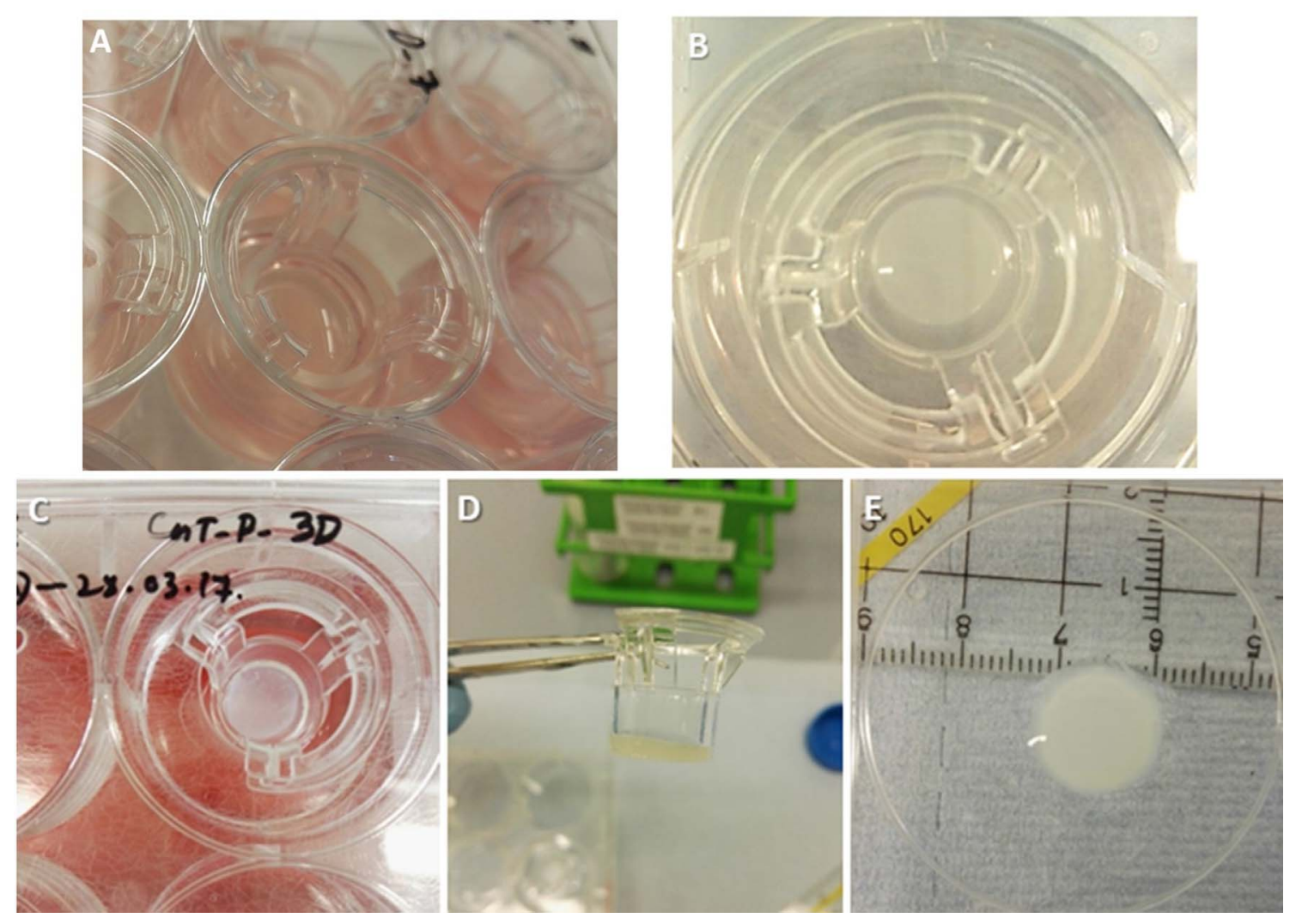

Fig. 2. Macroscopic view of Human Dermal Construct (HDC) - upper panel: (A) View of experimental set-up of Human Dermal Construct on Day-01 with NHDFs are embedded in collagen I matrix and incubated to allow gel remodeling. (B) Macroscopic top view of contracted collagen on Day-07 retaining its circular shape. Macroscopic view of HSE - lower panel: (C) Top view of experimental set-up of HSE development during incubation at ALI showing the white epidermal layer over the dermal layer. (D) Side view of HSE containing insert at the time of tissue harvesting after 20 days at ALI, showing a thickness of $\sim 2$ mm. (E) Top view of harvested HSE at the end of ALI period (20 days) retaining the size and diameter of initial mould (12 $\mathrm{mm}$ in diameter).

Table 2. Experimental set-up details of different 3D cell culture conditions (3D-CCs).

\begin{tabular}{|c|c|c|c|c|c|}
\hline Step duration & $\begin{array}{l}\text { Description of 3D culture } \\
\text { condition }\end{array}$ & 3D-CC-I & 3D-CC-II & 3D-CC-III & 3D-CC-IV \\
\hline 5-7 days & HDC feeding & FGM2 & FGM2 & $\begin{array}{l}\text { CnT-PR-F } \\
\text { (Medium A) }\end{array}$ & $\begin{array}{l}\text { CnT-PR-F } \\
\text { (Medium A) }\end{array}$ \\
\hline $3-4$ days & $\begin{array}{l}\text { Epidermal monolayer } \\
\text { formation (onto HDC); feeding }\end{array}$ & KGM2 & KGM2 & $\begin{array}{c}\text { CnT-PR } \\
\text { (Medium B) }\end{array}$ & CnT-PR-FTAL \\
\hline $15-16 \mathrm{~h}$ & $\begin{array}{l}\text { Epidermal monolayer } \\
\text { formation (onto HDC); feeding }\end{array}$ & - & $\begin{array}{l}\text { CnT-PR-3D } \\
\text { (Medium C) }\end{array}$ & $\begin{array}{l}\text { CnT-PR-3D } \\
\text { (Medium C) }\end{array}$ & - \\
\hline
\end{tabular}

Abbreviations: CnT-PR-F named as Medium A; CnT-PR named as Medium B; CnT-PR-3D named as Medium C.

\section{Immunophenotypic analysis}

HSE was rinsed in phosphate buffered saline (PBS) twice, treated with $2 \mathrm{M}$ sucrose solution (Fisher Chemical) at $4{ }^{\circ} \mathrm{C}$ for at least $1 \mathrm{~h}$ and then infiltrated in OCT (Optimal Cutting Temperature) embedding medium (VWR) for 20-30 min at room temperature (RT). The tissue was embedded in OCT by gradual freezing in liquid nitrogen vapor, stored at $-80^{\circ} \mathrm{C}$ and cryo-sectioned $(\sim 10 \mu \mathrm{m})$ using Microm HM 550 Cryostat.
Immunophenotypic analysis of epidermal markers was performed on cryosections. Specifications on the primary antibodies' concentrations used in this study are described in Table 3. Secondary antibodies used are the following: Alexa Fluor 488 Goat anti-Rabbit IgG $(\mathrm{H}+\mathrm{L})$ (Abcam), Alexa Fluor 594 Donkey anti-Mouse IgG $(\mathrm{H}+\mathrm{L})($ Abcam), Alexa Fluor 488 Donkey anti-Rabbit $\operatorname{IgG}(\mathrm{H}+\mathrm{L})$ (ThermoFischer), Alexa Fluor 568 Goat anti-Mouse IgG $(\mathrm{H}+\mathrm{L})$ (ThermoFischer). Negative controls were performed using only secondary antibodies. Cell nuclei were 
Table 3. Primary antibodies used for immunostaining of HSE tissue cryosections.

\begin{tabular}{lcc}
\hline Primary antibody details and source & Dilution & Clone-No. \\
\hline Ki-67 (Rb, MA) - Cell signal & $1: 300$ & D2H10 \\
Laminin 5 (Rb, PA) - Abcam & $1: 500$ & - \\
Keratin 14 (Ms, MA) - Abcam & $1: 100$ & LL002 \\
Keratin 10 (Rb, MA) - Abcam & $1: 500$ & EP1607IHCY \\
Filaggrin (Rb, PA) - Abcam & $1: 200$ & - \\
$\beta$-Tubulin (Ms, MA) - Sigma & $1: 400$ & EPR16774 \\
Loricrin (Rb, PA) - Abcam & $1: 200$ & - \\
Keratin 16 (Rb, PA) - Abcam & $1: 100 \& 2^{\circ} \mathrm{Ab}$ as $1: 200$ & - \\
\hline
\end{tabular}

$\mathrm{PA}=$ polyclonal antibodies; $\mathrm{MA}=$ monoclonal antibodies; $\mathrm{Rb}=$ rabbit; $\mathrm{Ms}=$ mouse; $2^{\circ} \mathrm{Ab}=$ secondary antibodies.

Table 4. Details of reagents required for Immunostaining.

\begin{tabular}{|c|c|c|}
\hline Reagent Type & Reagent constitution & Reagent Quantity (calculated) \\
\hline Fixative & $4 \%$ paraformaldehyde in PBS & $10 \mathrm{~mL}$ of $16 \%$ PFA in $30 \mathrm{~mL}$ PBS \\
\hline Antibody diluent buffer & $\mathrm{TBS}+1 \% \mathrm{BSA}$ & $0.1 \mathrm{~g} \mathrm{BSA}$ in $10 \mathrm{~mL}$ TBS \\
\hline Blocking Buffer & $\begin{array}{l}10 \% \text { normal serum }+1 \% \text { BSA }+ \text { TBS } \\
\text { For double staining: } 5 \% \text { normal serum of species in } \\
\text { which first secondary antibodies were raised }+5 \% \\
\text { normal serum of species in which second type of } \\
\text { secondary antibodies was raised }+1 \% \text { BSA }+ \text { TBS } \\
\text { Note: Goat and donkey serum } 60 \mathrm{mg} / \mathrm{mL}-\text { Jackson } \\
\text { Immuno Research }\end{array}$ & $1 \mathrm{~mL}$ normal serum $+0.1 \mathrm{~g} \mathrm{BSA}+9 \mathrm{~mL}$ TBS \\
\hline Buffers & $\begin{array}{l}1 \times \text { TBS }(10 \times \text { TBS comprised of } 500 \mathrm{mM} \text { tris } \\
\text { base }+1.5 \mathrm{M} \mathrm{NaCl}+\mathrm{HCl} \text { to set } \mathrm{pH} \text { to } 7.4) ; 1 \times \mathrm{PBS}\end{array}$ & $\begin{array}{l}100 \mathrm{~mL} \text { of } 10 \times \text { in } 900 \mathrm{~mL} \text { distilled deionized } \\
\mathrm{H}_{2} \mathrm{O}\end{array}$ \\
\hline
\end{tabular}

BSA is bovine serum albumin, TBS is tris-buffered saline, PBS is phosphate-buffered saline.

counterstained with DAPI $\left(4^{\prime}, 6\right.$-diamidino-2-phenylindole) $(1.5 \mu \mathrm{g} / \mathrm{mL}$, VECTASHIELD, Vector labs). Immunohistochemical (IHC) analysis was performed in accordance with the standard protocol from Abcam, with primary antibodies' concentrations optimized for HSE (Tab. 3). Details of reagents used in IHC staining are provided in Table 4. Cryosections were fixed in methanol-free $4 \%$ PFA (Thermo Scientific) for $10 \mathrm{~min}$ then rinsed in PBS (2 times for $5 \mathrm{~min}$ ). Sections were then permeabilized in $0.2 \%$ Triton X-100 for $10 \mathrm{~min}$ then rinsed in wash buffer (TBS, tris-buffered saline, $0.025 \%$ Triton X-100). Sections were blocked in blocking buffer $(10 \%$ normal serum from the same secondary antibody species, 1\% BSA, Bovine Serum Albumin, in TBS) for $2 \mathrm{~h}$ at room temperature. Slides were drained and treated with primary antibody diluted in antibody diluent buffer (1\% BSA in TBS) and incubated in a humidified chamber overnight at $4{ }^{\circ} \mathrm{C}$. Fluorophore-conjugated secondary antibody diluted in antibody diluent buffer were applied and incubated for $1 \mathrm{~h}$ at room temperature, protected from light. Finally, sections were rinsed in TBS (3 times for $5 \mathrm{~min}$ ) and mounted using VECTASHIELD mounting medium. The immunostained slides were examined with fluorescent microscope (Olympus XM10 with cellSense Standard software).

The percentage of Ki-67 positive dermal fibroblasts and basal keratinocytes was calculated from three IHC images $(n=3$ means three cryosectioned slices from a single
HSE, technical replicates and $N=2$, number of independent experiments) by dividing the Ki-67 positive nuclei (green) by total number of nuclei (blue). The equation applied is as follows: [N(Ki-67 positive cell nuclei)/ $N_{\text {total }}(\Sigma($ DAPI-stained cell nuclei, Ki-67 positive cells $\left.))\right] \times$ $100=\%$ (Ki-67 positive cells).

\section{Electron microscopy analyses: SEM and TEM}

Samples were fixed in $2.5 \%$ glutaraldehyde for $1 \mathrm{~h}$, washed in PBS (3 times for $5 \mathrm{~min}$ ), incubated with 1\% osmium tetraoxide for $15 \mathrm{~min}$, followed by PBS washing (3 times for $5 \mathrm{~min}$ ). Then, samples were dehydrated in an increasing gradient series of ethanol (30\%-5 min, 50\%$5 \mathrm{~min}, 70 \%$-overnight at $4{ }^{\circ} \mathrm{C}, 96 \%-5 \mathrm{~min}$ for 3 times, 100\%-15 min for 3 times) and submerged in polypropylene oxide (PO) for $15 \mathrm{~min}$ before inclusion in epoxy resin-based embedding medium (Epoxy Embedding Medium kit cat.-no. 45359, Sigma-Aldrich) with PO (1:1) for $1 \mathrm{~h}$ [38]. Thereafter, samples were embedded in the above mentioned medium in the right orientation and incubated at $80{ }^{\circ} \mathrm{C}$ for $8 \mathrm{~h}$. Tissue blocks were ultrasectioned ( $40 \mathrm{~nm})$ using Leica Ultracut UCT Ultramicrotome, contrasted with Leica ultrastain 2 (3\% lead citrate) and Leica ultrastain $(0.5 \%$ uranyl acetate), and imaged with JEM-2100 TEM at an accelerating voltage of $200 \mathrm{kV}$. 
For scanning electron microscopy (SEM), samples were fixed and dehydrated as described above. Then, samples were placed in BAL-TEC CPD 030 Critical Point Dryer and dryed with liquid $\mathrm{CO}_{2}$ by short consecutive immersions (3 times for $10 \mathrm{~min}$ ). Then, samples were mounted onto electrically conductive, double sided adhesive carbon discs (Leit C tabs) and gold coated using Edwards S150B Sputter Coater under Argon gas $\left(10^{-1} \mathrm{mbar}\right)$ at voltage of $15 \mathrm{~mA}$ for $6 \mathrm{~min}$ to form $40 \mathrm{~nm}$ thick gold $(\mathrm{Au})$ coating. Coated samples were examined with SEM DSM 982 Gemini, Zeiss. Multiple SEM images were taken at random areas of different samples at $15 \mathrm{kV}$ accelerating voltage. The diameter of the collagen fibers was estimated as an average value from multiple TEM images.

\section{Wettability analysis}

Static contact angle measurements were performed to investigate wettability of HSE by sessile-drop method, using custom-built contact angle goniometer instrument under ambient conditions. An amount of $5 \mu \mathrm{L}$ MilliQ water was carefully placed on dry HSE prepared in two different experiments $(N=2$; Number of independent experiments); Multiple images were recorded (shown as an example in Supplementary Fig. 1S) and analyzed for 10 drops per HSE surface $(N=2 ; n=10)$.

\section{Statistical analysis}

All experiments, where not explicitly described otherwise, were performed independently in duplicate $(N=2$, number of independent experiments) and 3 technical replicates were made and examined per experiment $(n=3)$. Standard deviations were given where appropriate and expressed as mean $\pm \mathrm{SD}$ (standard deviation). For statistical analysis, GraphPad Prism 5.00.288 (Inc., San Diego, CA, USA) was used to evaluate the significance of the differences in experimental data. Significance between groups was considered for $p<0.05$.

\section{Results}

The development of dermal-epidermal based HSE was optimized to obtain well-differentiated epidermis onto HDC. To this purpose, different 3D-CCs based on cell culture media and the parameter of ALI (Tab. 2) were applied showing significant impact on tissue morphogenesis.

\section{Tissue morphogenesis of HSCs under different 3D-CCs}

Figure 3 shows histological images of HSCs obtained using 3D-CC-I and 3D-CC-II, respectively. Two structurally distinct compartments epidermis and dermis were present. Epidermis was slightly better organized in 3D-CC-II (Figs. 3, $2 \mathrm{~A}-2 \mathrm{D}, 2 \mathrm{~A}^{\prime}-2 \mathrm{D}^{\prime}$ ) than in 3D-CC-I (Figs 3; 1A-1C, $\left.1 \mathrm{~A}^{\prime}-1 \mathrm{C}^{\prime}\right)$, which on the contrary displayed larger gaps between the layers. When comparing both culture conditions, it is noticeable that the stratum corneum (SC) in the case of 3D-CC-II forms a clearer or more coherent layer. Furthermore, organization of epidermal layers under 3D-CC-II significantly improved when air-lift (ALI) culture

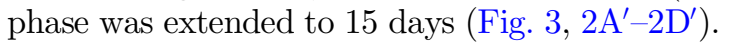

Furthermore, compared to 3D-CC-IV (based on CnT-FTAL, Fig. 4), 3D-CC-III (based on CnT-PR-3D (Medium C), Fig. 5) demonstrated an improved differentiated epidermal formation.

Among the tested conditions, improved morphogenesis results were obtained under 3D-CC-III and at ALI for 20 days, resulting in constructs better mimicking epithelial differentiation found in healthy human skin. Hence, such HSC was termed as human skin equivalent (HSE) (Figs. 5A" $-5 \mathrm{D}^{\prime \prime}$ ). The epidermal part showed improved differentiated layers of keratinocytes namely stratum basale (SB), spinosum (SS), granulosum (SG), and corneum (SC) (Fig. 6A), more comparable with its in vivo counterpart (Fig. 6B).

\section{IHC analysis of differentiation markers on HSE}

IHC characterization of HSE (Figs. 7.1A and 7.1B) showed the presence of specific epidermal differentiation markers. Cytokeratin-14 (K14) was expressed by epithelial cells in the SB (Fig. 7.1A), while the suprabasal layers displayed the presence of Cytokeratin-10 (K10) (Fig. 7.1B). Cells in basal layer were found positive for Ki67 (highlighted by the arrows), demonstrating an active state of proliferation (Fig. 7.2E). After counting, HSE displayed $10.5 \pm 0.8 \%(p$-value $<0.05) \mathrm{Ki}-67$ positive basal keratinocytes. The terminal differentiation of epidermis was demonstrated by the spotted expression (highlighted by arrows) of filaggrin (Flg) in the SG (Fig. 7.1C). Loricrin (Lor), a major protein component of cornified envelope and marker of terminally differentiated epidermal cells, also showed spotted expression in SC (highlighted by arrows in Fig. 7.2F). Involucrin (Inv) was displayed in upper spinous, sub-corneal, and corneal layers (Fig. 7.2G). Laminin 5 (Lam 5) was expressed at DEJ as a thin continuous line (Fig. 7.1D) suggesting stable epidermal-dermal interaction. Beta-tubulin as a component of the cytoskeletal microtubules was mainly found as a continuous seam in the SB area, where differentiating and proliferating NHEK are located, and in the NHDF in the HDC area (Fig. 7.2E).

\section{Comparison of immunoreactivity among different 3D-CCs}

IHC analysis for cytokeratins showed that K14 and K10 were expressed by epithelial cells in SB (Fig. 8A) and suprabasal layers (Fig. 8B) of HSC obtained under 3D-CC-III and at ALI for 15 days. However, K14 and K10 expression was lower than for HSE obtained under 3D-CC-III and at ALI for 20 days (Figs. 7.1A and 7.1B).

Similarly, IHC analysis for cytokeratins was performed on HSC obtained from 3D-CC-IV at ALI for 20 days. Results showed that K14 and K10 were expressed by epithelial cells in the SB (Fig. 9A) and suprabasal layers 

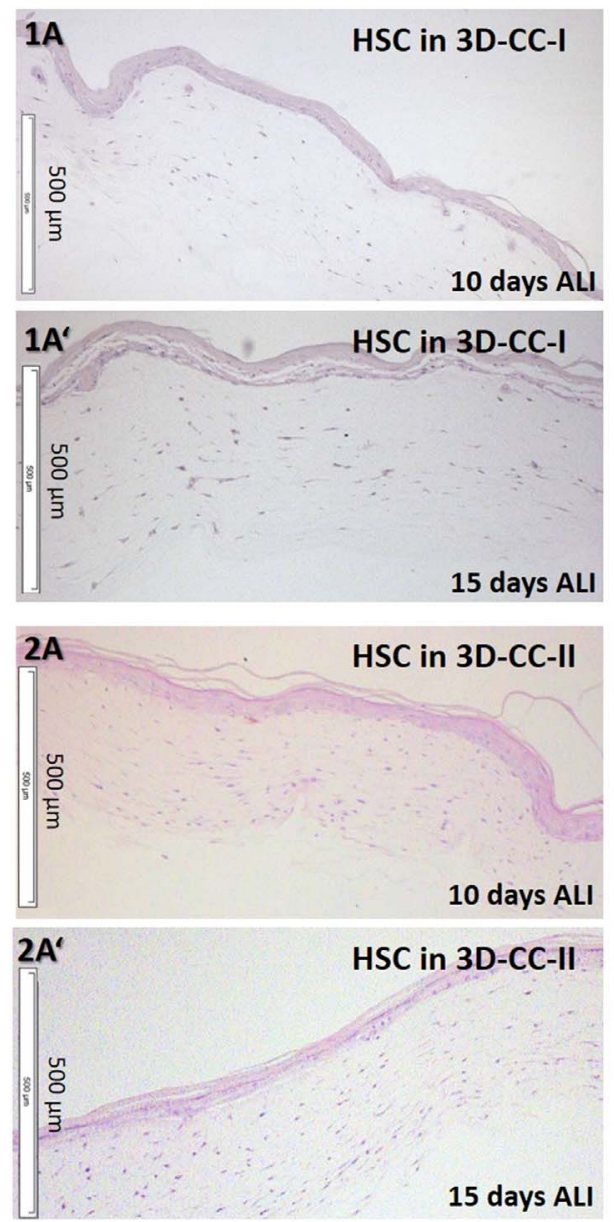
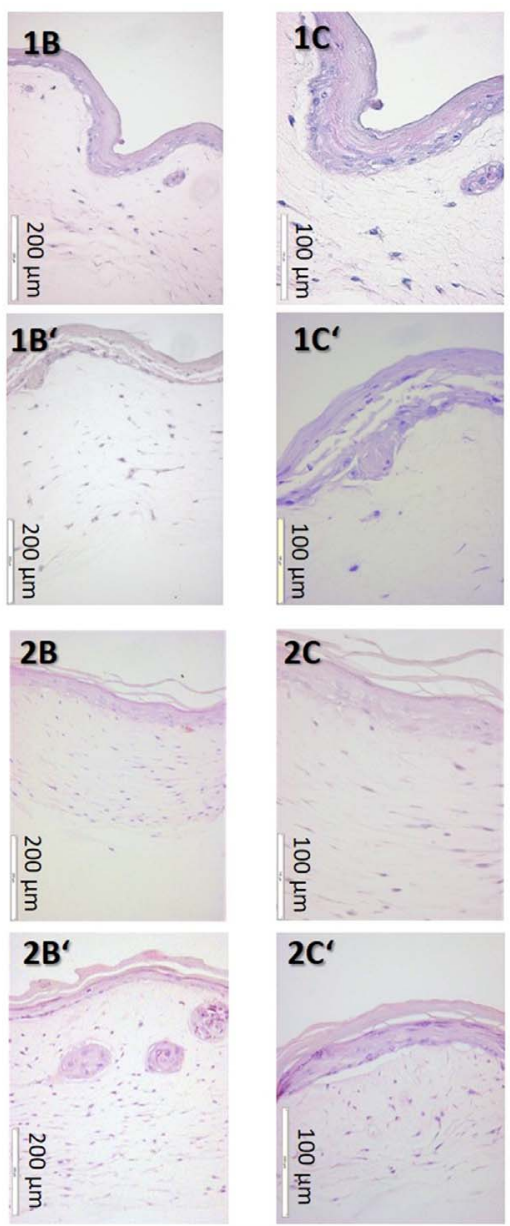
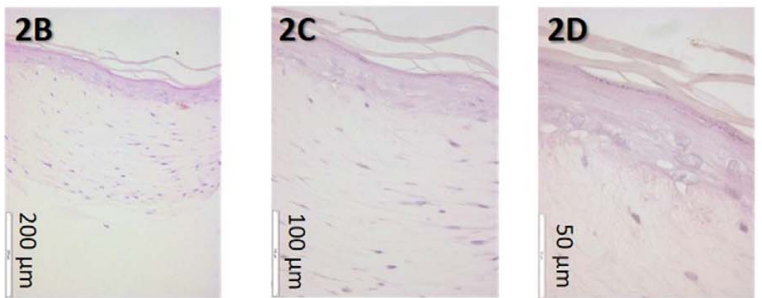

$2 D^{4}$

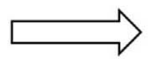

Fig. 3. Histological cross sections of HSC using the $3 \mathrm{D}$ culture conditions $3 \mathrm{D}-\mathrm{CC}-\mathrm{I}$ (upper panels $1 \mathrm{~A}-\mathrm{C}$ and $1 \mathrm{~A}^{\prime}-\mathrm{C}^{\prime}$ ) and $3 \mathrm{D}-\mathrm{CC}-\mathrm{II}$ (lower panels $2 \mathrm{~A}-\mathrm{D}$ and $\left.2 \mathrm{~A}^{\prime}-\mathrm{D}^{\prime}\right) ; N=2, n=3$. Upper panels $(1 \mathrm{~A}, 1 \mathrm{~B}, 1 \mathrm{C})$ and $\left(1 \mathrm{~A}^{\prime}, 1 \mathrm{~B}^{\prime}, 1 \mathrm{C}^{\prime}\right)$ showed H\&E-stained histological microscopy images of HSCs at different magnification elevated to the air-liquid interface (ALI) for 10 days and 15 days, respectively. Lower panels $(2 \mathrm{~A}, 2 \mathrm{~B}, 2 \mathrm{C}, 2 \mathrm{D})$ and $\left(2 \mathrm{~A}^{\prime}, 2 \mathrm{~B}^{\prime}, 2 \mathrm{C}^{\prime}, 2 \mathrm{D}^{\prime}\right)$ show H\&E-stained histological microscopy images of HSCs at different magnification elevated to the air-liquid interface (ALI) for 10 days and 15 days, respectively.

(Fig. 9B), respectively. However, K14 and K10 expression was found comparatively lower than for HSE (3D-CC-III, at ALI for 20 days) (Figs. $7.1 \mathrm{~A}$ and 7.1B), while Ki-67 was found similarly expressed (Fig. 9C) as in HSE (Fig. 7.2E).

\section{Ultrastructure (TEM) of HSE}

TEM was used to investigate the ultrastructure of HSE. Regarding the dermal compartment, TEM analysis confirmed the presence of embedded fibroblasts in a network of collagen- and immature elastin-based ECM (Fig. 10D). Collagen fibrils combine with each other to form collagen fibers. In HSE, collagen formed both fibers and fibrils (Figs. 10A-10D) with the characteristic banded pattern with a period of $\sim 65 \mathrm{~nm}[39,40]$. Elastin was found deposited on collagen fibrils or microfibrils in an amorphous immature form (Fig. 10C) [41].

Figures $10 \mathrm{~A}$ and $10 \mathrm{~B}$ also show hemidesmosomes connecting basal cells through tonofibrils/keratin filaments and attaching them into dense basal lamina through anchoring filaments. On the other side, Figures $10 \mathrm{E}$ and $10 \mathrm{H}$ show an abundance of keratins and tonofilaments in keratinocytes, and the presence of tight junctions and lipid droplets in SG. TEM imaging finally also demonstrated the presence of desmosomes as spot-like connections for cell-tocell adhesion of keratinocytes (Figs. 10F-10H).

\section{Tissue architecture (SEM) of HSE}

Histological analyses highlighted the typical elongated morphology of NHDF (Fig. 6A). SEM images confirmed the presence of embedded fibroblasts, with dendritic extensions lying along dense matrix of collagen fibers (Fig. 11B). ECM of the dermal compartment of HSE demonstrated the typical interwoven network morphology and architecture (Fig. 11A).

The average diameters of microfibril, collagen fibril, and collagen fiber calculated from multiple TEM images $(n=3)$ were $26 \pm 21 \mathrm{~nm}, 65 \pm 57 \mathrm{~nm}$, and $157 \pm 10 \mathrm{~nm}$ 

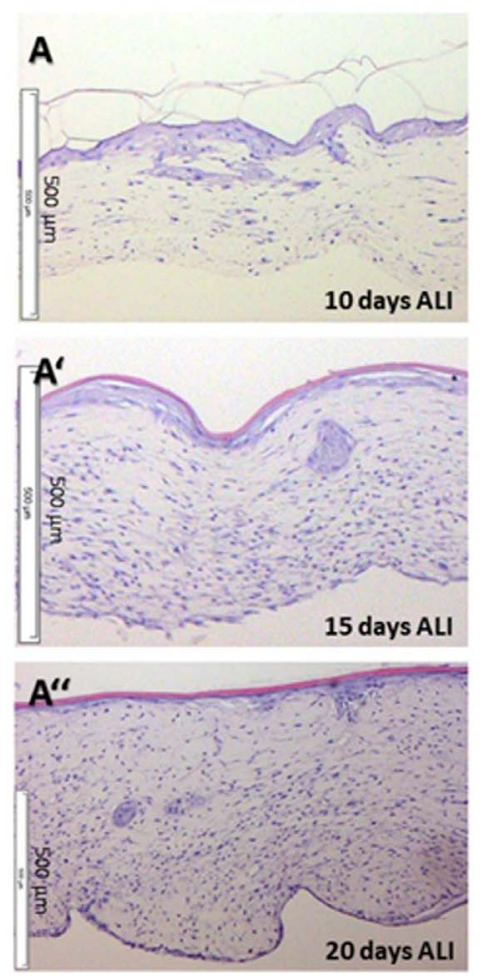
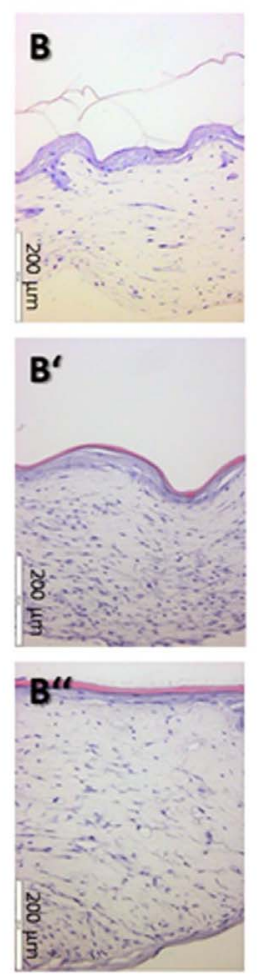
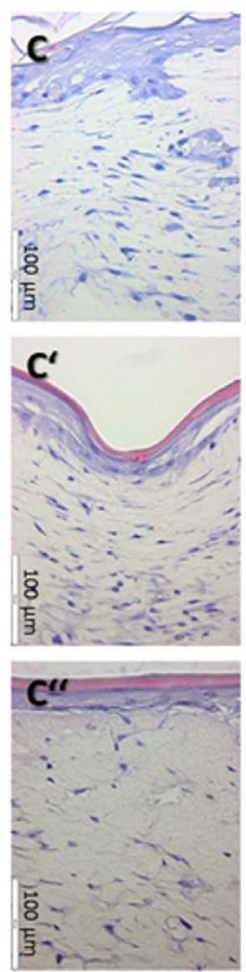
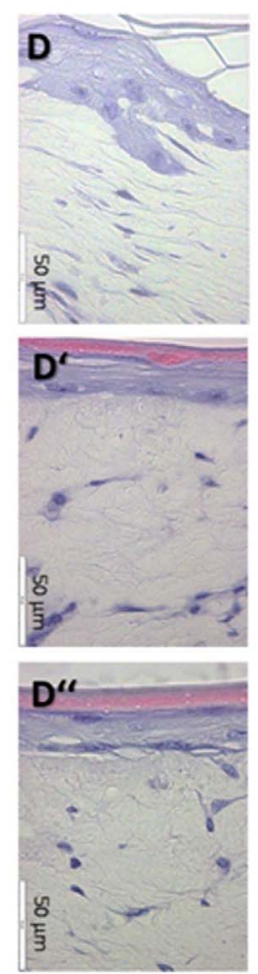

Fig. 4. Histological cross sections of HSC using the $3 d$ culture conditions $3 \mathrm{D}-\mathrm{CC}-\mathrm{IV} ; \mathrm{N}=2, \mathrm{n}=3$. Upper (A, B, C, D), middle (A', $\mathrm{B}^{\prime}$, $\left.\mathrm{C}^{\prime}, \mathrm{D}^{\prime}\right)$, and lower panels $\left(\mathrm{A}^{\prime \prime}, \mathrm{B}^{\prime \prime}, \mathrm{C}^{\prime \prime}, \mathrm{D}^{\prime \prime}\right)$ (images at different magnifications) showed H\&E-stained histological micrographs of HSCs raised for 10 days, 15 days, and 20 days at ALI, respectively. The longer the ALI cultivation period, the better a structured stratification of the epidermis from stratum basale to stratum corneum can be recognised (cf. labelling in Fig. 6).
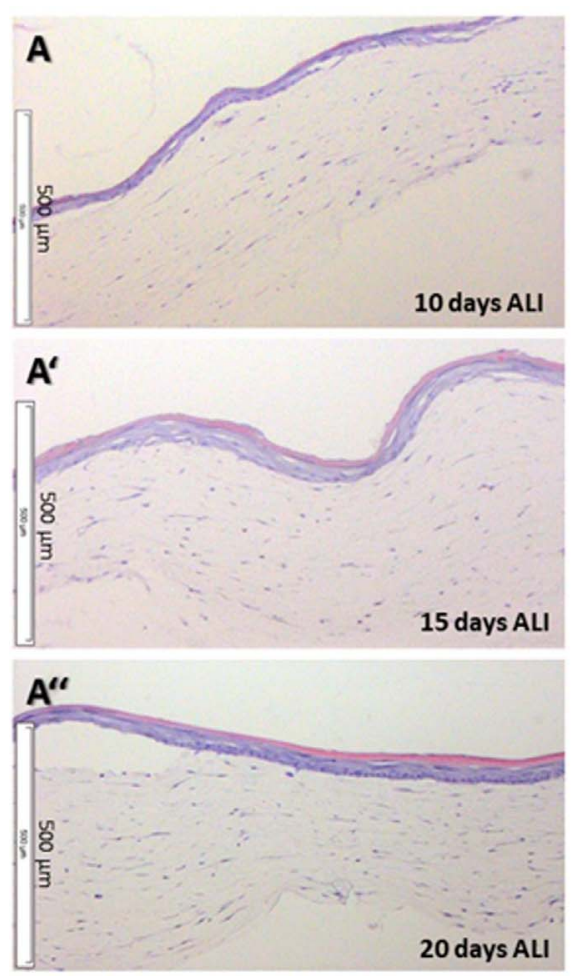
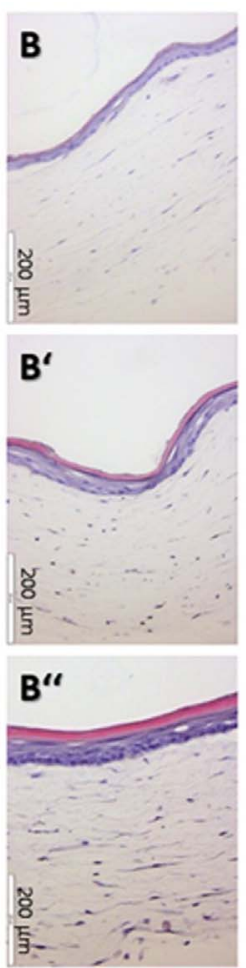
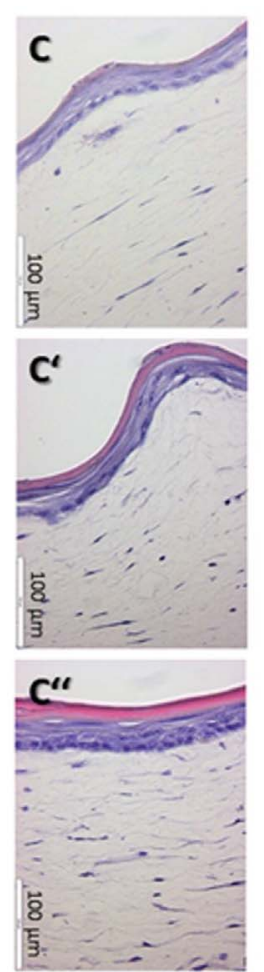
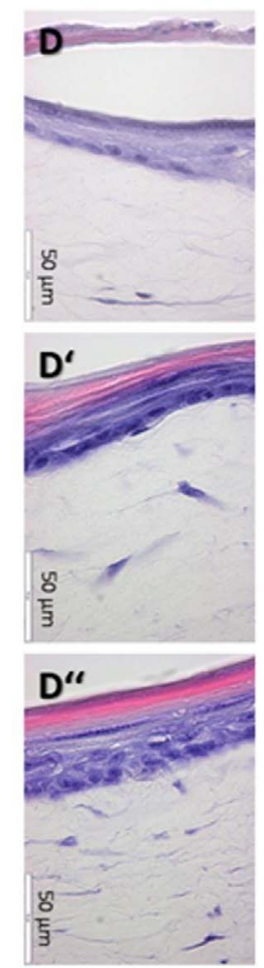

Fig. 5. Histological cross sections of HSC using $3 \mathrm{D}$ culture condition $3 \mathrm{D}-\mathrm{CC}-\mathrm{III} ; N=2, n=3$. Upper (A, B, C, D), middle (A', B', C', $\left.\mathrm{D}^{\prime}\right)$, and lower $\left(\mathrm{A}^{\prime \prime}, \mathrm{B}^{\prime \prime}, \mathrm{C}^{\prime \prime}, \mathrm{D}^{\prime \prime}\right)$ panels (images at different magnifications) showed H\&E-stained histological micrographs of HSCs raised for 10 days, 15 days and 20 days at ALI, respectively. The longer the ALI cultivation period, the better a structured stratification of the epidermis from stratum basale to stratum corneum can be recognised (cf. labelling in Fig. 6). 

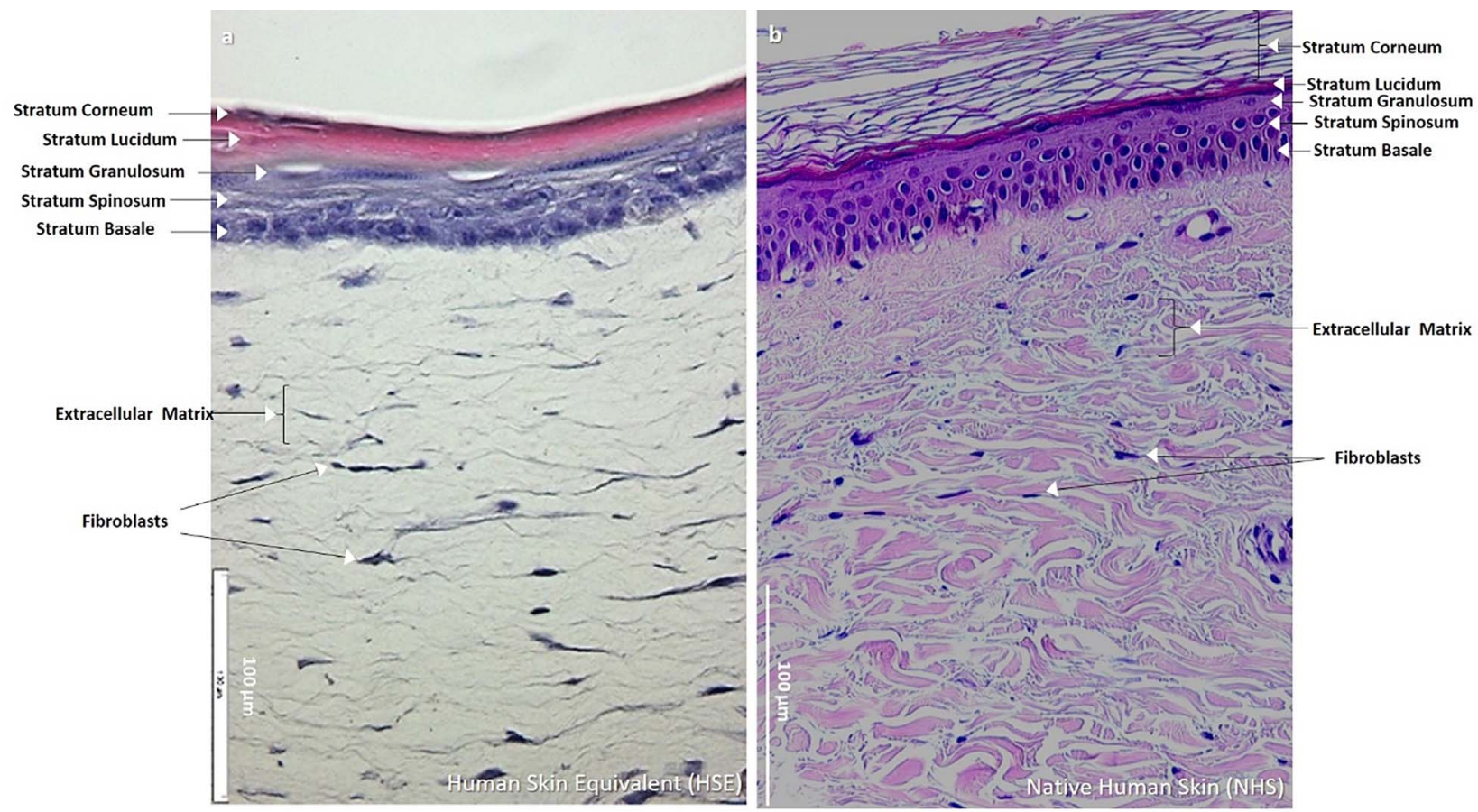

Fig. 6. (a) HSC as HSE. H\&E-stained histological image of HSC obtained from 3D-CC-III (cf. Fig. 5C'") was able to best recapitulate epidermal differentiation, morphogenesis and organization similarly as found in (b) NHS (origin: human leg; histology: different staining protocol) and thus named as HSE. HSE showed two structurally distinct layers of skin the outer epidermal layer, and the underlying thicker dermal layer. The epidermal part showed well differentiated layers of keratinocytes namely stratum basale, spinosum, granulosum, and corneum. The staining differences between HSE and NHS are due to a different H\&E protocol used to stain NHS at the Institute of Pathology, Ruhr University Bochum, Germany, where the NHS tissue was obtained.
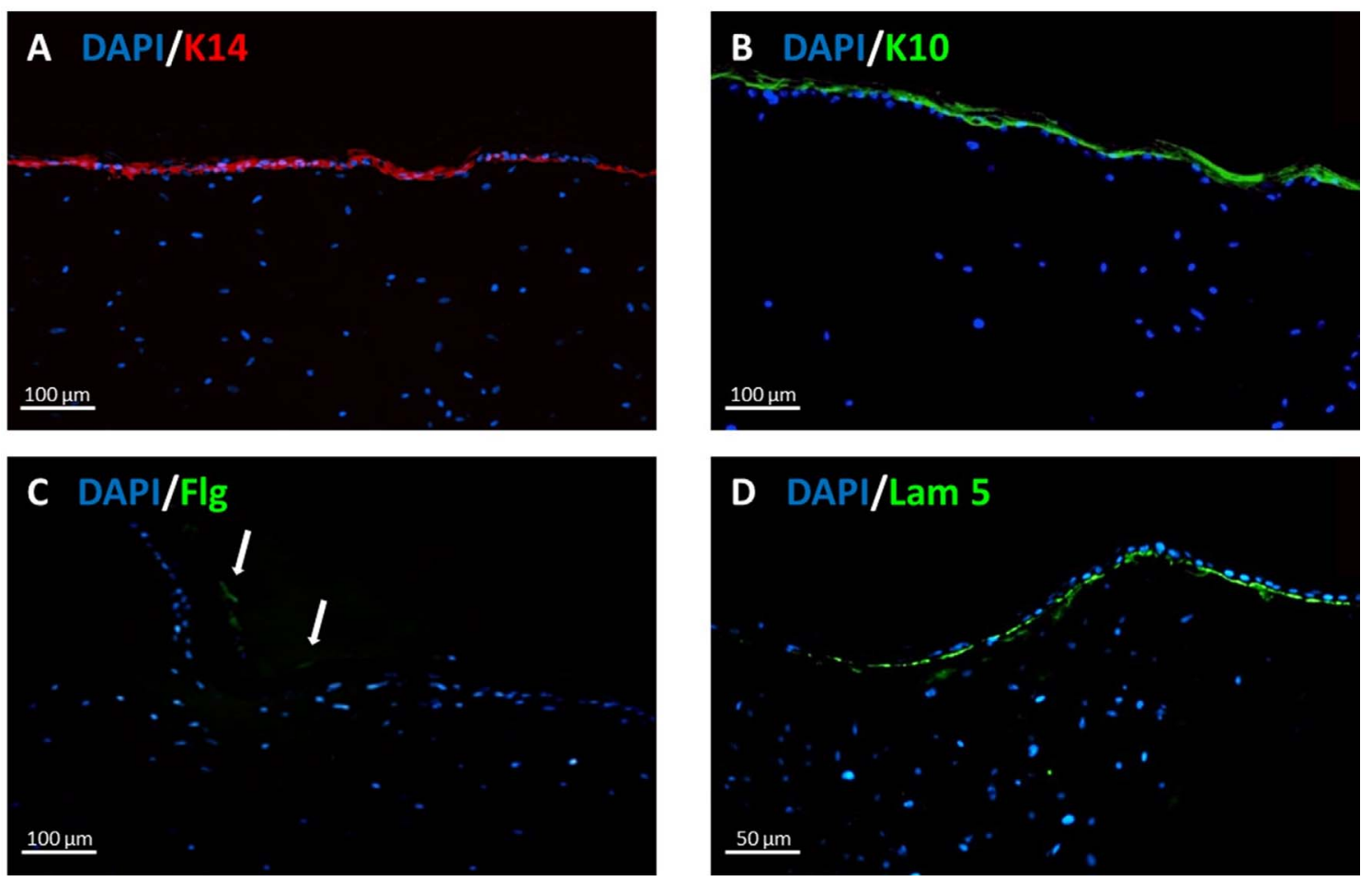

Fig. 7.1. Immunotypic analysis of HSE. IHC characterization of HSE showed immunolabelling for specific epidermal differentiation markers namely (A) Keratin 14 (K14) red, (B) Keratin 10 (K10) green, (C) Filaggrin (Flg) green (indicated by white arrows), and (D) Laminin 5 (Lam 5) green. Cell nuclei were counterstained with DAPI (in blue); $N=2, n=3$. 

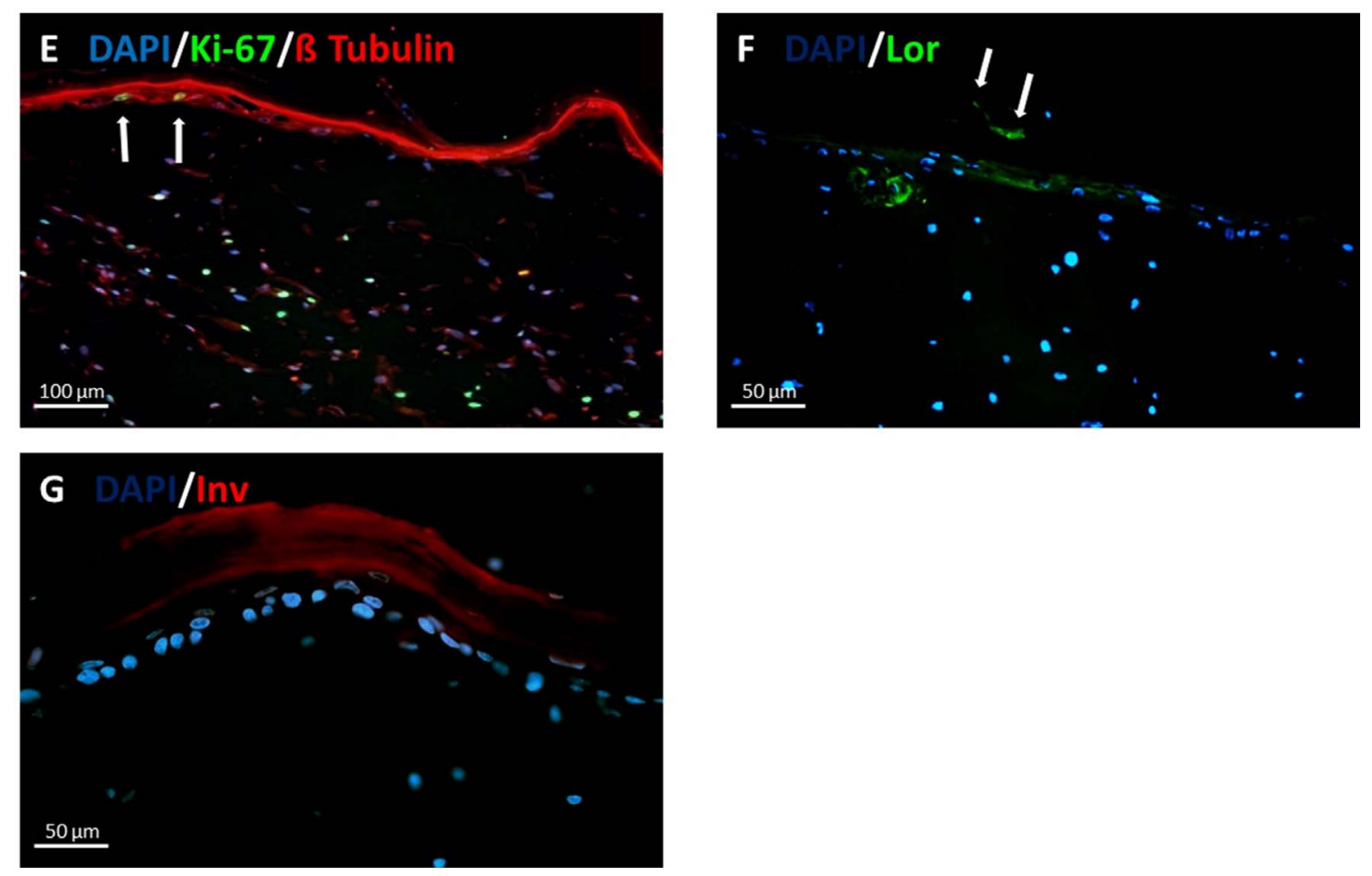

Fig. 7.2. Further immunotypic analysis of HSE. (E) Detection of proliferative keratinocytes in the area of the stratum basale of the epidermis (white arrows) and of division-active fibroblasts in the dermis by using the proliferation marker Ki-67 green, further evidence for specific epidermal differentiation was provided by the detection of (F) Loricrin (Lor) green, and ( $\mathrm{G}$ ) Involucrin (Inv). Cell nuclei were counterstained with DAPI (in blue) and microtubules in cytoplasm were shown in red using $\beta$ Tubulin staing (E); $N=2, n=3$.

respectively. Fibril-associated molecules on the surface of collagen fibrils play an important role in controlling interactions between the fibrils, and thus the relative frequency of collagen bundles' diameters. ECM showed thinner collagen fibers than mature collagen bundles, suggesting an immature state of the dermal compartment [42,43].

SEM images also showed adjacent stratified epidermal cells when approaching SC (Figs. 11C-11F). NHEK of basal and suprabasal epidermal layers showed a typical morphology and were continuously organized (Fig. 11C). When keratinocytes are cultured in vitro to develop 3D tissue, one of the main issues is the limited longevity of keratinocytes due to the long culture time at ALI (e.g. $2-3$ weeks). The surface layers of developed epidermis become very thick because of their inability to desquamate causing the compression of the lower layers, as observed in SEM results (Figs. 11C-11D). In vivo, the normal desquamation occurs unnoticeably: mature keratinocytes move from SB to SG in 14 days, then they are shed off [44].

\section{Barrier function properties of HSE}

An important interest associated with well-developed $\mathrm{SC}$ is the skin barrier function. This functionality was evaluated by measuring the surface wettability of HSE that is highly related with physiological surface properties of skin. The static Water Contact Angle (WCA) value of HSE was
$82.5 \pm 8.9^{\circ}$ that was highly comparable (difference not significant at $p$-value $<0.05)$ to the value $\left(90.0 \pm 5.1^{\circ}\right)$ reported for NHS [45]. This result is in agreement with results obtained by TEM and SEM analysis (Figs 10 and 11) demonstrating that SC had formed, providing protection with tight cell junctions (corneo-desmosomes), lipids sealing intercellular spaces, and the presence of keratin filaments (including filaggrin and degradation products) (Figs. 7.1C, 7.2F, 7.2G).

\section{Dermal compartment of HSE}

IHC was performed for laminin expression in dermal compartment of HSE and was detected as fainted spots around the fibroblasts (Fig. 12). Moreover, the percentage of Ki-67 positive dermal fibroblasts calculated was $24.7 \pm 7.8 \%$, indicating their proliferative potential.

\section{Discussion}

\section{Development of dermal layer of HSE}

Connective tissue has an important role as a substrate for the growth of epidermal tissue. Particularly ECM proteins affect skin tissue phenotype [46]. For instance, the use of decellularized dermis, e.g. AlloDerm (a commercially available cadaveric dermis for clinical applications) favors the fast assembly of basement membrane [33]. The 

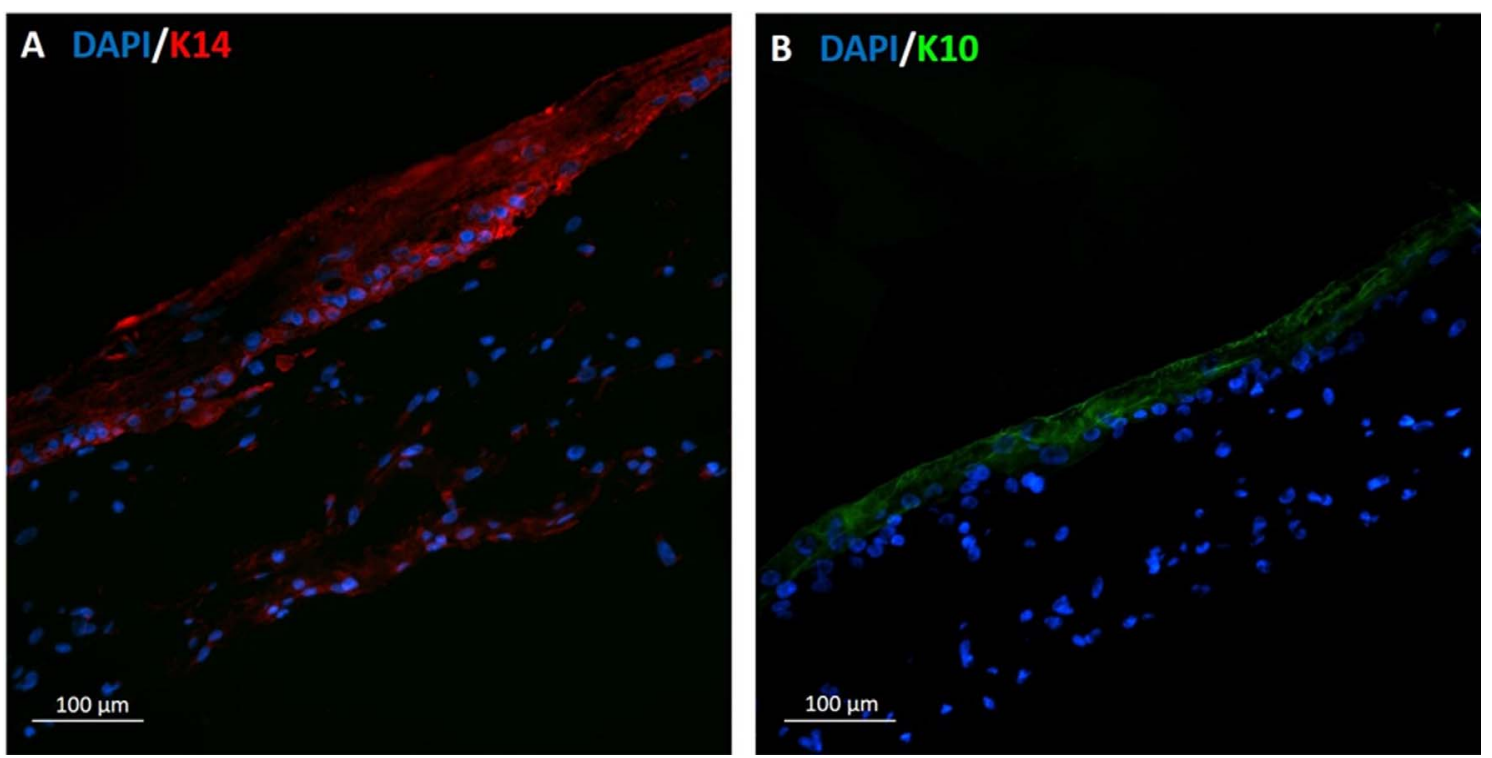

Fig. 8. Immunotypic analysis of HSC using 3D-CC-III at 15 days of ALI. IHC analysis of HSE showed immunolabelling for specific epidermal differentiation markers, namely (A) Keratin 14 (K14) red, and (B) Keratin 10 (K10) green. Cell nuclei were shown in blue by using DAPI staining; $N=2, n=3$.
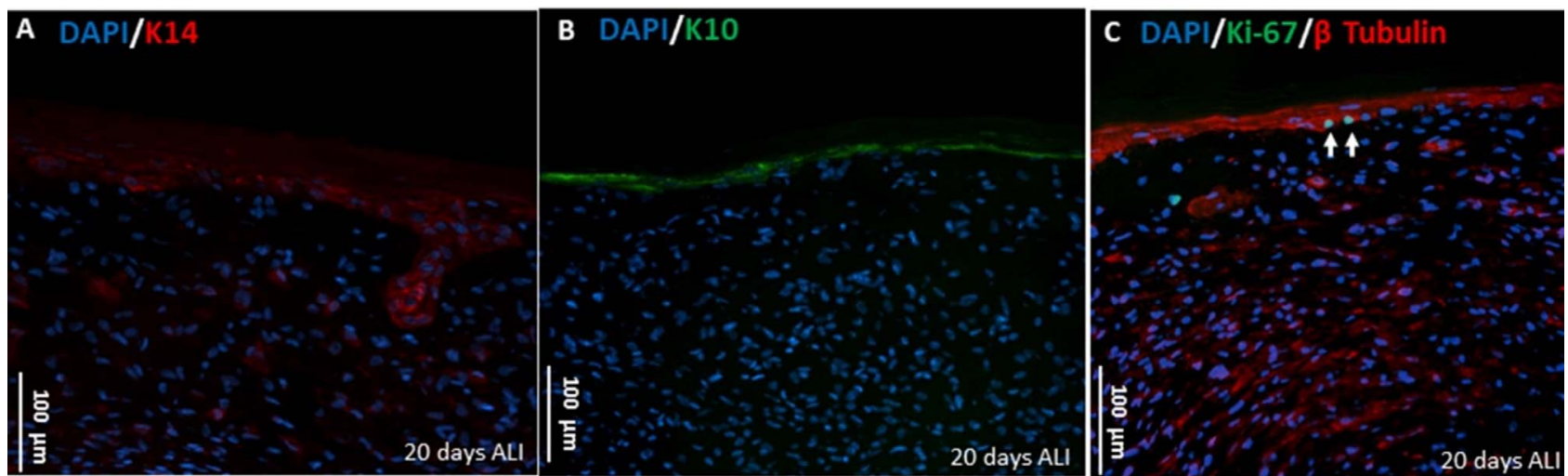

Fig. 9. Immunotypic analysis of HSC using 3D-CC-IV. IHC analysis of HSE showed immunolabelling for specific epidermal differentiation markers namely (A) Keratin 14 (K14) red, (B) Keratin 10 (K10) green, and (C) Ki-67 green. Cell nuclei were counterstained with DAPI (in blue) and microtubule in cytoplasm keratinocytes and fibroblasts are shown in red using $\beta$ Tubulin staining (C). Exemplary presentation of the quantification of Ki-67 positive cells: [N(Ki-67 positive cell nuclei) $/ N_{\text {total }}(\Sigma(\mathrm{DAPI}-$ stained cell nuclei, Ki-67 positive cells) $)] \times 100=\%$ (Ki-67 positive cells), $[(2 / 19) \times 100=10.5 \%$ (in relation to the image section being viewed); $N=2, n=3$.

contracted collagen gel used in this work might result in a less intact basement membrane, however it greatly supported keratinocyte growth and differentiation.

In this work, a 3D functional HSE was prepared by successively fabricating the dermal and epidermal compartments (Fig. 1). Optimal culture conditions for the engineering of a biomimetic HSE were: (i) NHDF $\left(\sim 8 \times 10^{4}\right.$ cells $\left./ \mathrm{mL}\right)$ culture within Col. I gel for $5-7$ days in CnT-PR-F medium; ii) NHEK $\left(8 \times 10^{6}\right.$ cells $\left./ \mathrm{mL}\right)$ seeding on the top of dermal layer and culture in submerged conditions for 3 days using CnT-PR medium; iii) submerged culture overnight in CnT-PR-3D medium to stimulate the formation of cell-cell contacts; iv) culture of epidermal layer at ALI for 15-20 days while keeping dermal construct in submerged culture conditions using CnT-PR3D medium.

\section{Epidermal differentiation of HSE}

HDC was previously developed based on Col. I from rat tail tendons, mimicking skin ECM thanks to its irregular fibrils with in vivo-like structure [47]. The development of HSE with dermal-epidermal compartments has been improved by using commercially available primary cells and ready-to-use media provided according to Good Laboratory Practice (GLP) and Good Manufacturing Practice (GMP) criteria to form a well-differentiated epidermis accessible to everyone. To this purpose, the effect 

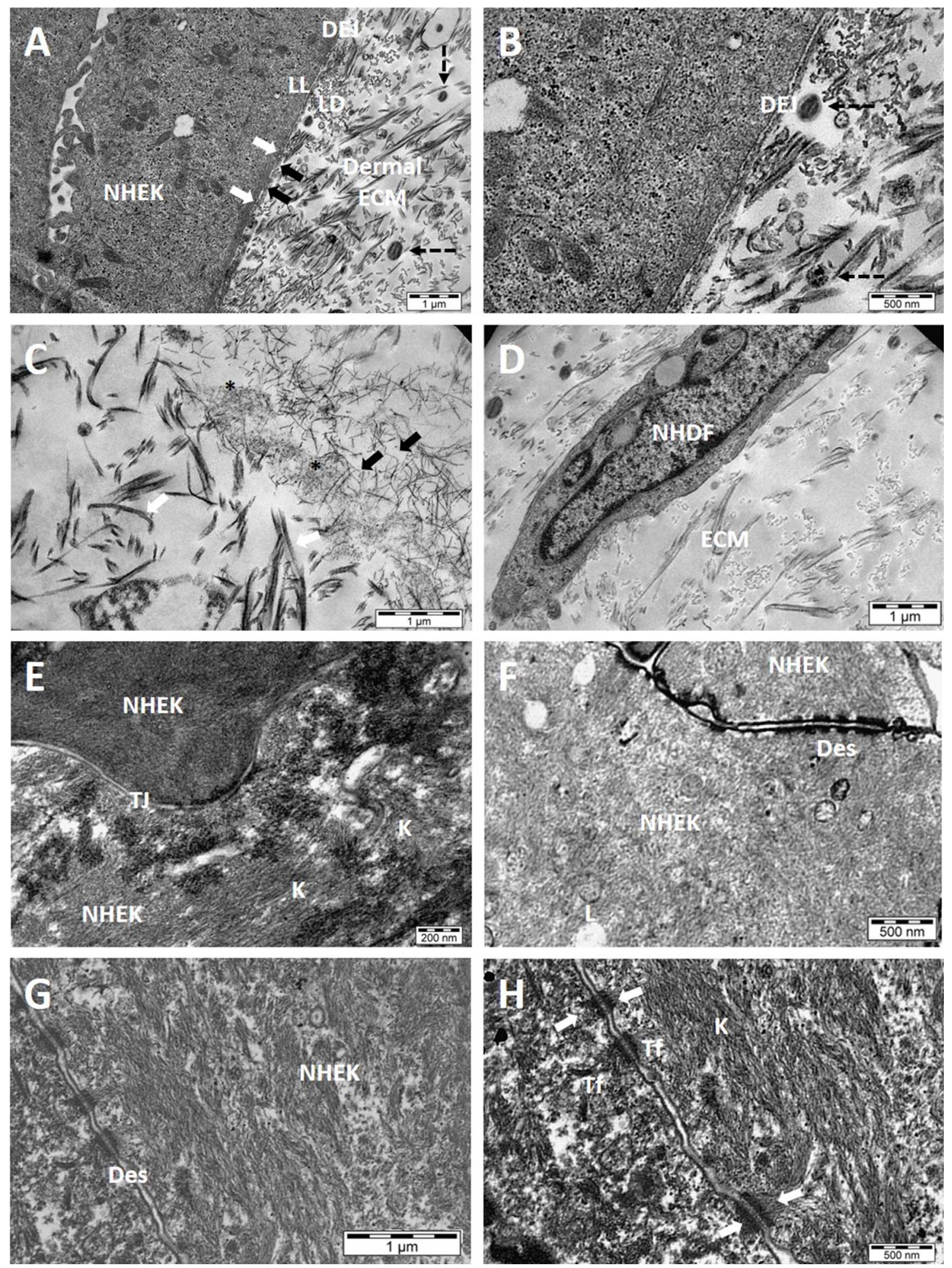

Fig. 10. Ultrastructure analysis of HSE. TEM images (A, B) revealed dermal-epidermal junction (DEJ) that separates epidermis from the dermis through basement membrane consisting of lamina lucida (LL) and lamina densa (LD). LD was connected with the collagen matrix by loops of anchoring fibrils (black arrows). The hemidesmosomes (white arrows) were connected with tonofibrils (Tf) in keratinocytes. Collagen fibers were shown as black dotted arrows. TEM images (C, D) demonstrated collagen fibrils (Col. I) with their characteristic banded pattern (white arrows). Microfibrils or fibrillins (black arrows) were also secreted by fibroblasts, forming a scaffold that was detected near collagen fibrils. Amorphous or "immature" elastin was secreted and aggregated (asterisks) on microfibrils. Collagen fibers (black dotted arrow) appeared when collagen fibrils combined to form fibers. Normal Human Dermal Fibroblasts (NHDF) were also seen as embedded in ECM. In TEM images (E, F, G, H) epidermis showed tight junction (TJ) between the keratinocytes. Keratinocytes showed abundant intracellular filament of keratins (K) and upper epidermal layers showed lipid droplets (L). Tonofibrils (Tf) in keratinocytes (NHEK) were made of tonofilaments (keratin intermediate filaments), that appear converging to form spot like connections or desmosomes (Des or white arrows); $N=2, n=3$. 

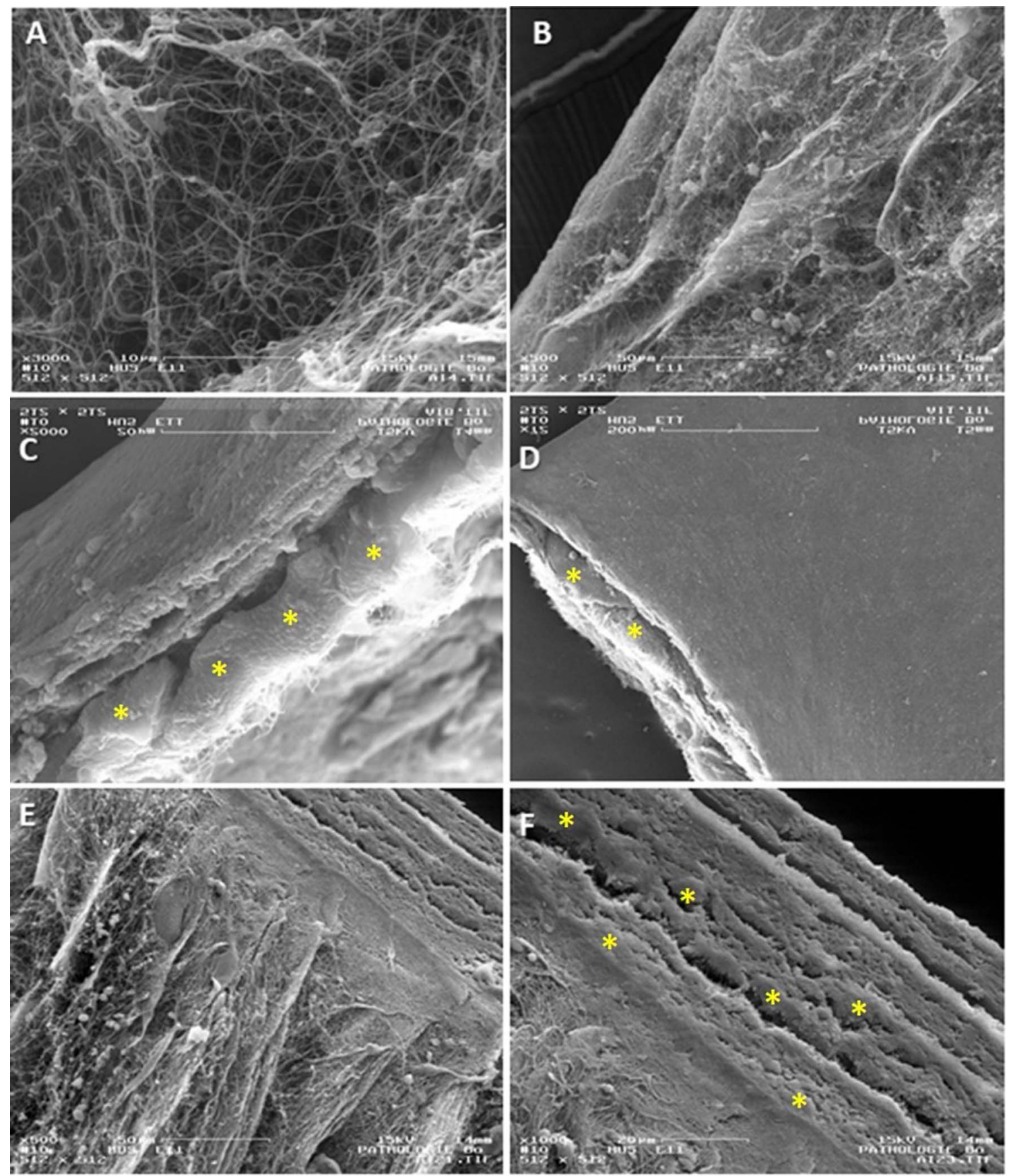

Fig. 11. SEM analysis of HSE. (A, B) SEM images of dermal compartment of HSE showed ECM with a typical interwoven network morphology and architecture with embedded dermal fibroblasts lying along dense matrix of collagen fibers. (C, D, E, F) Epidermal layers of skin were increasingly flattened as they move to the surface to form SC. The inner layers of keratinocytes (C) were continuous with less flattened morphology. Keratinocytes of the individual layers are marked with yellow asterisk; $N=2, n=3$.

of different 3D-CCs (Tab. 2) on tissue morphogenesis was analyzed.

It is known that calcium activates intracellular signaling able to initiate epidermal differentiation [48]. For this reason, media constituted or commercially available for 3D culture at ALI were supplemented with a high calcium concentration $(1.2 \mathrm{mM})$. The role of ALI in epidermal differentiation has been attributed to the formation of calcium gradient in the epidermal compartment (due to water loss in the higher cell layers) is achieved due to air-lift [49]. ALI also helps in achieving the proper lipid profile (acylglucosylceramides and acylceramides) of SC [50], as the rate of trans-epidermal water loss influences lipid biosynthesis [51]. Moreover, cells cultured at ALI are not in direct contact with cell culture medium components, such as retinoids, that strongly inhibit epidermal differentiation [52].

Growth factors have also an important role in epidermal differentiation. The traditional culture media used to 


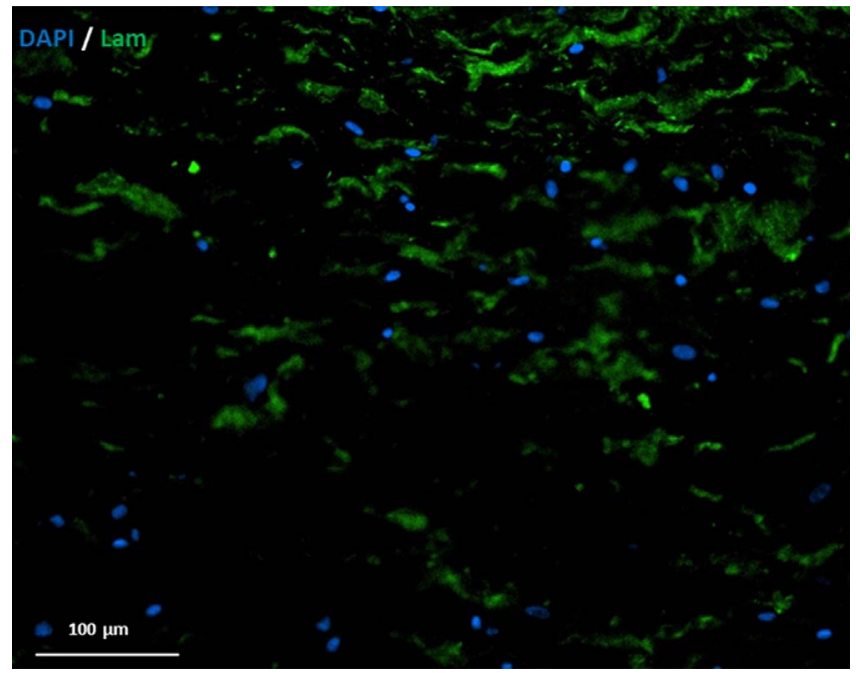

Fig. 12. ECM proteins in dermal compartment. IHC analysis of HSE showed immunolabelling for one of the ECM proteins laminin in green. Cell nuclei are shown in blue (DAPI staining); $N=2, n=3$.

expand biopsies from human amniotic membrane include hydrocortisone, triiodothyronine, adenine, and cholera toxin [53-56]. Hydrocortisone has significant role for maintaining epithelial colonies and keratinocyte proliferation [57]. Triiodothyronine, being a supplement hormone, has been shown to play a beneficial role in keratinocyte cultivation under minimal levels of fetal calf serum [58]. Cholera toxin, a protein secreted by Vibrio cholerae that causes cholera infection (watery diarrhea) has been shown to highly stimulate colony growth of cultured human epidermal keratinocytes through increased levels of intracellular cyclic adenosine monophosphate [59]. Adenine also has an effect on epithelial cells by improving their colony forming ability [60-62]. Among the several stimuli inducing keratinocyte differentiation, ascorbic acid has been reported to cause an increased expression of the genes encoding cornified envelope proteins. The pro-differentiating effects of ascorbic acid follow a similar signaling pathway as the ones mediated by calcium. However ascorbic acid-associated differentiation is also accompanied by an enhanced ascorbate transport and a prevention of hydrophilic antioxidants depletion $[63$, 64].

Additionally, in the optimization of 3D-CCs for the development of HSE, the commonly used self-constituted cornification media for 3D skin culture are not animal component-free. In fact, they contain bovine calf serum (BCS) or fetal calf serum (FCS) and $\mathrm{CaCl}_{2}$, along with the above mentioned vital elements [65, 66]. Besides causing challenging safety issues for clinical applications, serum presence at ALI has been reported to impair the terminal differentiation of keratinocytes [67].

In this work, a gradual shift towards the use of animal component-free cell culture media to optimize HSE development was performed. To this aim, the effects of four different 3D-CCs (-I, -II, -III, -IV) on tissue morphogenesis were analyzed (Tab. 2).
ALI period was optimized by comparing the tissue morphogenesis of HSCs obtained from different air-lift periods of 10,15 , and 20 days.

KGM2 (PromoCell), a serum-free medium containing $0.06 \mathrm{mM} \mathrm{CaCl} \mathrm{Cl}_{2}$ was used to culture keratinocytes at submerged conditions (3D-CC-I) and was then supplemented with $\mathrm{CaCl}_{2}$ to achieve a concentration of $1.2 \mathrm{mM}$ during air-lift phase.

CnT-Prime media are fully-defined, animal componentfree and furthermore without cholera toxin. The basal CnT medium, such as CnT-PR medium is supplemented with $\mathrm{CaCl}_{2}$ (0.07 mM concentration), progenitor cell targeted (PCT) growth factors able to extend cell longevity, and co-factors able to improve growth factor binding to membrane-bound receptors. On the other hand, the CnT prime media for 3D culture, such as CnT-PR-3D and CnT-FTAL, contain elevated levels of calcium ions $(1.2 \mathrm{mM})$, while they do not contain PCT growth factors, or other proliferative factors that retard differentiation, thus providing maximal stimulus to differentiation and increased barrier function.

Figure 3 shows histological images of HSCs obtained using 3D-CC-I and 3D-CC-II. Epidermis was comparatively better organized in 3D-CC-II compared to 3D-CC-I, which instead displayed larger gaps between the layers (Figs. 3, 1A-1C, 1 $\mathrm{A}^{\prime}-\mathrm{C}^{\prime}$ ). Among the examples of abnormal or pathological epidermal conditions, formation of cell nests in dermis depicts malignant behavior of melanocytes [68], as it happens in early tumor invasion in vivo with the melanoma cells getting into dermis and invading the basement membrane [69]. Another example is the formation of thickened scaly skin plaques due to hyperplasia, which is an indication of psoriatic skin and is histologically detected through spongiform micro-pustules and elongated rete ridges [70]. The presence of wide interlayer spaces is one of the abnormalities of skin appearance, to be avoided through improved tissue morphogenesis, in order to mimic healthy skin structure.

Under 3D-CC-II (Figs. 3, 2A-2D, 2A'-2D'), the epidermal layers were better organized after 15 days of air-lift compared to 10 days condition. Exposure to ALI enabled keratinocytes to differentiate and recapitulate the morphological as well as biochemical processes of native human epidermis [71, 72]. Hence, cell cultures for extended periods at ALI comparatively demonstrated improved morphological and biochemical differentiation, structural organization,

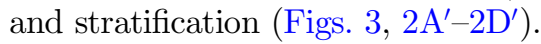

Figures 4 and 5 show histological images of HSCs obtained from 3D-CC-IV (based on CnT-FTAL) and 3DCC-III (based on CnT-PR-3D), respectively. CnT-PR-3D medium is recommended for the development of 3D "epidermal model" at air-lift phase, while CnT-FTAL medium is recommended to be used during the air-lift phase of "dermal-epidermal full-thickness skin model" to promote ECM secretion by fibroblasts. The work showed better organized and differentiated epidermal tissue, after 20 days culture at air-lift, under 3D-CC-III using CnT-PR-3D medium, rather than under 3D-CC-IV, using CnT-FTAL medium. 


\section{Protein distribution in HSE}

The sequential expression of cytokeratins allowed to follow epidermal differentiation.

In the skin, K5 and K14 are expressed in the mitotically active cells in SB. When these cells leave the SB and enter the differentiation program, they become postmitotic, downregulating K14 mRNAs expression and switching to expression of another set of keratins from K5/K14 to suprabasal keratins K1/K10 in the SS. Such changes in keratin expression pattern (and keratin pair expression) are particularly important as they confer precise functional requirements to epidermal keratinocytes [73]. The expression of differentiation-specific keratins causes keratin filament network reorganization resulting in denser bundle formation [74]. In more detail, cells from inner layers of the epidermis have small keratins $(46-58 \mathrm{kDa})$, while cells from the outer layers contain large keratins $(63-67 \mathrm{kDa})$ [75]. These changes in keratin composition and synthesis occur during the course of terminal differentiation. Keratins on the outermost layer have to be synthesized earlier, in order to be post-translationally processed during the final phases of differentiation, considering there is no synthesis in SC [75]. In our model, K14 and K10 keratins, are shown to be respectively expressed in basal and suprabasal layers (Figs. 7.1A, 7.1B), as support of the differentiation programme described above. Ki-67, a cellular marker for proliferation, is present during all active phasesf of the cell cycle and is absent in quiescent $\left(\mathrm{G}_{0}\right)$ cells. The Ki-67 antigen could be exclusively detected within the cell nucleus, which demonstrated a proliferative state of basal keratinocytes in HSE (Fig. 7.2E). Moreover, HSE displayed 10.5\% Ki-67 positive basal keratinocytes, comparable to the in vivo proliferation rate of $10-12 \%$ measured by Ki-67 expression in the stratum basale of NHS [76].

Cells in SS also produce involucrin, a cornified envelope component, and transglutaminase-K, the enzyme responsible for crosslinking of involucrin in SC [77, 78]. SG has electron-dense keratohyalin granules containing profilaggrin (precursor of filaggrin), and the smaller granules contain loricrin, that is a major constituent of cornified envelope $[79,80]$. During terminal differentiation of epithelial cells, filaggrin is post-translationally produced from large profilaggrin precursor protein. Filaggrin is a filament-aggregating protein, that connects keratin fibers by promoting disulfide-bond formation among the intermediate filaments. The terminal differentiation of epidermis was demonstrated by the expression of Involucrin, loricrin, and filaggrin (Figs. 7.1C and 7.2F, 7.2G).

The stable attachment of the epidermis to the dermis was shown by DEJ presence, suggesting the formation of basement membrane. The epidermal basal cells connected to papillary dermis through anchoring filaments of hemidesmosomes. The proteins within the anchoring complex provided links to both the intracellular cytoskeletal keratins in keratinocytes and connective tissue proteins of the papillary dermis. Laminins, a family of ECM glycoproteins are major non-collagenous components of basement membranes. Laminin 5 is a key component of this anchoring complex that initiates hemidesmosome formation in the basal lamina, and has also an important role in accelerating the assembly of basement membranes as well as enhancing the recovery of damaged skin [81]. Laminin 5 expression at DEJ demonstrated a stable epidermal-dermal interaction (Fig. 7.1D).

Moreover, HSE revealed the typical ultrastructure features, tissue architecture, and physiological barrier function properties of human skin. In Figures 10A and 10B, the connection between epidermis and DEJ interface was demonstrated by the presence of hemidesmosomes, connecting basal cells through tonofibrils/keratin filaments and attaching them into dense basal lamina through anchoring filaments. Basal lamina is one of the two layers of basement membrane and is further divided into lamina lucida (LL) and lamina densa (LD) [67]. Regarding the connection of dermis through DEJ, loops of fibrils perpendicularly arranged to basal lamina have been reported to anchor into DEJ. They are called oxytalan elastic fibers and their presence suggests a strong cohesion between DEJ and dermal compartment [41].

On the other side, in Figures 10E-10H, the epidermal compartment showed an abundance of keratins and tonofilaments in keratinocytes, tight junctions, and lipid droplets in SG. In vivo, SG contains lamellar bodies and lipid-containing vesicles (as observed in Fig. 10F) that secrete lipid content into the connection between SG and $\mathrm{SC}$ [82].

The matured outermost layer (SC) is a cornified envelope around bundled keratin filaments which is adherent to the lipid envelope contributed by the SG, resulting in an impermeable covering protecting the lower layers [83]. Results also demonstrated the presence of desmosomes as spot-like connections for cell-to-cell adhesion of keratinocytes, which in vivo enable skin to bear intense mechanical stresses.

Altogether, these findings demonstrated complex ECM network organization (even if still immature) of HSE dermal compartment, epidermal differentiation, and DEJ maturation.

\section{Protein expression of HSCs under different 3D-CCs}

Immunotypic analysis for K14 and K10 (Fig. 8 compared to Figs. 7.1A, 7.1B) demonstrated differences in epidermal differentiation under 3D-CC-III at air-lift for " 15 days" vs. "20 days". K14 and K10 expression was lower at air-lift for "20 days" than for " 15 days". These findings indicated the positive role of "longer air-lift phase" on tissue morphogenesis and differentiation.

Immunotypic analysis for K14, K10, and $\beta$-Tubulin (Fig. 9 compared to Figs. 7.1A, 7.1B, and 7.2E) demonstrated the differences in epidermal differentiation in HSC under 3D-CC-IV vs. 3D-CC-III. K14, K10, and $\beta$-Tubulin expression was lower under 3D-CC-IV than under 3D-CC-III, confirming that the culture conditions had an impact on tissue morphogenesis, on protein expression, and on microtubules degradation and reorganisation in corneocytes of SC [84]. 
Normal and abnormal expression of epidermal markers is highly related with the physiological and pathological conditions of skin. For example, a "skin blistering disorder" namely bullous congenital ichthyosiform erythroderma (BCIE) or epidermolytic hyperkeratosis (EHK) is caused by defects in KRT10 gene and histologically seen as diffused epidermolytic degeneration of lower spinous layer in epidermis [85]. Other "skin blistering conditions" involving intraepidermal epidermolysis bullosa named epidermolysis bullosa simplex Dowling-Meara type (DM-EBS), WeberCockayne type (WC-EBS), Koebner type (K-EBS), and autosomal recessive (AREBS) are caused by defects in KRT14 [86]. Defects in loricrin cause a skin condition with "erythematous plaques" named as progressive symmetric erythrokeratodermia (PSEK) and Vohwinkel syndrome with ichthyosis (VSI) [87]. Therefore, the right level of protein expression at correct anatomical location is important for defining the morphological and functional characteristics of skin tissue. In our model, morphological analysis of histological cross sections (Fig. 6A) and IHC results (Figs. 7.1, 7.2) confirmed the proper selection of HSC obtained from "3D-CC-III" at air-lift for "20 days" as "HSE" for a good differentiation toward full-thickness skin phenotype.

\section{Dermal fibroblasts of HSE}

Dermal ECM contains many components secreted by dermal fibroblasts including, collagen, elastin, laminin, fibronectin, and hyaluronic acid. Results showed that human dermal fibroblasts actively produced laminin (Fig. 12). Hence, cells provided a physiological environment by depositing ECM molecules.

Another important aspect in 3D cultures is the presence of a homeostatic equilibrium that depends on collagen remodeling as well as on proportion of proliferative vs. non-proliferative dermal fibroblasts $[88,89]$. Collagen remodeling equilibrium can be calculated as degree of collagen assembly indicating the assembled fraction of collagen network. In this study, IHC analysis allowed measurement of dermal fibroblast immunoreactivity for Ki-67 as an indication of their active proliferation status. The percentage of Ki-67 positive dermal fibroblasts was $24.7 \pm 7.8 \%$, similar to values reported in the literature for an endogenous human dermal equivalent $(24.9 \pm 6.2 \%)$ [45], thus suggesting a similar proliferative potential.

The proliferative behavior of fibroblasts explains the non-contraction behavior of HSE during epidermis formation. It was experimentally observed that HSE retained its size and shape during epidermal development on the top of HDC (Figs. 2B and 2E).

\section{Clinical applications of HSE}

There are currently some commercially available temporary or permanent skin substitutes with approval for medical use in patients. A distinction is made between epidermal, dermal and dermal-epidermal skin substitutes
(DESS). In the case of extensive full-thickness skin defects (FTSD), as can occur in burns, avulsion injuries, septic skin necrosis or iatrogenic defects, autologous split-thickness or full-thickness skin transplantation is nevertheless currently the gold standard among surgical defect coverage concepts. Healthy skin (usually in the form of split skin) is removed from one part of the patient's body, creating a secondary defect, and transplanted onto the defect site. CUTISS AG (a Swiss Company) has developed a method or process to generate an autologous DESS with the product name denovoSkin $^{\mathrm{TM}}$ from a single skin biopsy. This DESS is a personalised bio-engineered skin substitute that has now been registered as an Advanced Therapeutic Medicinal Product (ATMP) and is being tested in initial clinical trials (phase 1 and 2b). To the best of our knowledge, technological details have not been published at present.

At present, it is not foreseeable whether such a medical product will be affordable for any health system. The HSE developed as part of this work is a first step towards establishing an open-source platform that is accessible to everyone, can be reproduced in any cell culture laboratory in the world and can be further developed for various applications in research and clinical settings.

\section{Conclusions}

A dermal-epidermal organotypic model of human skin equivalent (HSE) was developed by optimizing the 3D cell culture conditions, in order to: (1) guide the cells towards the formation of a well-differentiated and fully functional epidermal tissue and (2) ensure the correct cross-talk signals between dermis and epidermis. Histological results of HSE showed characteristic multi-layered epidermis with well-differentiated layers, while immunostaining analysis supported the expression of dermal and epidermal markers at accurate anatomical locations. TEM and SEM revealed ultrastructure features and tissue architecture, respectively. Static WCA analysis demonstrated the barrier function of HSE.

The here developed HSE may represent a suitable in vitro model for investigating pathophysiological events involving dermis-epidermis interactions. In this regard, HSE also provides a reliable pre-clinical testing platform for the in vitro screening of novel biomaterials, therapeutics or cosmetics. Alternatively, HSE could be used as allogenic or autologous skin graft (depending on the cell source) to fulfill the demand for skin graft, e.g. in the treatment of burnt patients.

This model represents a open-source, fundamental basic construct with potentialities for further improvements by the substitution of the here still used rat tail collagen type I by human recombinant Col. I and by enclosure of advanced skin features: for example, the addition of Langerhans cells would help this model to serve as a tool for studying skin immunological reactions, while the introduction of skin appendages would help to conduct more reliable drugs/cosmetics penetration studies. 


\section{Nomenclature}

$3 \mathrm{D}$

3D-CCs

ALI

AREBS

ATMP

$\mathrm{Au}$

$\beta$

BCIE

BCS

BSA

BSS

CnT-PR

CnT-PR-3D

CnT-PR-F

CnT-PR-FTAL CELLnTEC-Prime-full thickness airlift

Col. I

DAPI

DEJ

DESS

DM-EBS

ECM

EDTA

EGF

EHK

EM

FCS

bFGF

FGM2

Flg

FTSD

GLP

GMP

HEPES

$(\mathrm{H}+\mathrm{L})$

HSCs

HSE

$\operatorname{IgG}$

IHC

IL-1

Inv

iPSCs

K1

K5

K10

K14

K-EBS

$\mathrm{KGF}$

KGM2

Three-dimensional

3D cell culture conditions

Air-liquid interface

Autosomal recessive epidermolysis

bullosa simplex

Advanced Therapeutic Medicinal

Product

Aurum (gold)

Beta

Bullous congenital ichthyosiform

erythroderma

Bovine calf serum

Bovine serum albumin

Buffered saline solution

CELLnTEC-Prime epithelial culture

CELLnTEC-Prime three-dimensional

barrier

CELLnTEC-Prime Fibroblast

Collagen type I

4',6-diamidino-2-phenylindole

Dermal-epidermal junction

Dermal-epidermal skin substitutes

Epidermolysis bullosa simplex Dowling-

Meara

Extracellular matrix

Ethylenediaminetetraacetic acid

Epidermal growth factor

Epidermolytic hyperkeratosis

Electron microscopy

Foetal calf serum

Basic fibroblast growth factor

Fibroblast Growth Medium 2

Filaggrin

Full-thickness skin defects

Good Laboratory Practice

Good Manufacturing Practice

(2-[4-(2-hydroxyethyl)piperazine-1-yl]

ethanesulfonic acid

Heavy chain and light chain

Human skin constructs

Human skin equivalent

Immunoglobulin G

Immunohistochemistry

Interleukin-1

Involucrin

Induced pluripotent stem cells

Cytokeratin-1

Cytokeratin-5

Cytokeratin-10

Cytokeratin-14

Epidermolysis bullosa simplex Koebner

Keratinocyte growth factor

Keratinocyte growth medium 2

\begin{tabular}{ll} 
Ki-67 & Kiel 67 \\
Lam 5 & Laminin 5 \\
Lor & Loricrin \\
mM & Millimolar (mmol/L) \\
mRNA & Messenger ribonucleic acid \\
$N$ & Number of independent experiments \\
$n$ & Number of technical replicates \\
NaHCO & Sodium bicarbonate \\
NHDFs & Normal human dermal fibroblasts \\
NHEKs & Normal human epidermal keratinocytes \\
NHS & Native human skin \\
OCT & Optimal cutting temperature \\
PBS & Phosphate buffered saline \\
PCT & Progenitor cell targeted \\
PFA & Paraformaldehyde \\
PO & Polypropylene oxide \\
PSEK & Progressive symmetric erythrokeratoder- \\
& mia \\
RT & Room temperature \\
SD & Standard deviation \\
SEM & Scanning electron microscopy \\
SB & Stratum basale \\
SC & Stratum corneum \\
SG & Stratum granulosum \\
SS & Stratum spinosum \\
TBS & Tris-buffered saline \\
TEM & Transmission electron microscopy \\
TNS & Trypsin neutralizing solution \\
TBS & Tris-buffered saline \\
VSI & Vohwinkel syndrome with ichthyosis \\
WCA & Water contact angle \\
WC-EBS & Epidermolysis bullosa simplex \\
& Weber-Cockayne \\
& \\
\hline &
\end{tabular}

\section{Supplementary material}

Supplementary Material is available at https:// www.4open-sciences.org/10.1051/fopen/2021001/olm.

Supplement Figure 1S. Water contact angle (WCA) measurement: Water droplet was placed automatically via a Hamilton $\mu$-syringe on top of HSE. CA was measured after $60 \mathrm{sec} ; \mathrm{N}=2, \mathrm{n}=10$.

\section{Acknowledgments}

Authors acknowledge Ms. Wenfa Yu from the Faculty of Chemistry and Biochemistry, Analytical Chemistry Biointerfaces (Rosenhahn-Lab) at the RUHR-University Bochum, Germany for assisting with contact angle measurement facility.

\section{Conflict of interest}

The authors declare that the research was conducted in the absence of any commercial or financial relationships that could be construed as a potential conflict of interest. 


\section{Author contributions}

$\mathrm{AI}, \mathrm{VC}$, and JS contributed the conception and design of the study. AI performed all experiments and wrote the first manuscript draft. Data analysis and optimization of the protocols were carried out by AI and JS. VC and JS supervised the current research throughout, established the collaborations for making the analyses possible, revised, and approved the submitted manuscript version. IS provided important contributions for SEM \& TEM analysis and provided the scanning electron micrographs \& histological images of native human skin. SP takes the credit for conducting the basic cell culture training and envision of initial results. AZ contributed in the discussion, writing and revision of the manuscript. DG was involved in the creation and optimization of the figures, tables and in the image analysis evaluation.

He also did a linguistic revision. SS and RV provided clinically retrieved human-derived skin tissue samples for further histological processing and pathomorphological analysis. GC and RV, being two of the main HyMedPoly project partners contributed by facilitating the Project management for the conduction of current research.

\section{Funding}

This work was supported by the European Union's Horizon 2020 research and innovation program under the Marie Sklodowska-Curie Grant Agreement No. 643050 (HyMedPoly Project).

\section{Contribution to the field}

Human skin constructs are tissue-engineered skin equivalents with potential applications in regenerative medicine, and as in vitro models, allowing preclinical testing of new wound dressing biomaterials, therapies and studies on the pathophysiology of skin diseases. Since 2009, European regulation has forbidden the use of animals for cutaneous resorption tests - e.g. needed for cosmetics, paving the way towards alternative in vitro models. Hence, the present work has a key role in the framework of the 3Rs ("Replacement, Reduction and Refinement") principle, introduced by European regulation, aimed at replacing, reducing and refining the animal experimentation in research. Up to now, studies on in vitro engineered dermal-epidermal based skin models have been limited, while simple but poorly predictive epidermal models have been widely investigated. On the other hand, the use of freshly excised skin, as an advanced in vitro skin model, alternative to engineered human skin constructs, has been limited by poor availability and ethical constrains.

Based on that, this work was aimed at the in vitro engineering of an innovative dermal-epidermal human skin construct, recapitulating a similar organization and function of normal human skin, presenting huge potentialities for research and clinical purposes.

\section{References}

1. Wong T, Mcgrath JA, Navsaria H (2007), The role of fibroblasts in tissue engineering and regeneration. $\mathrm{Br} \mathrm{J}$ Dermatol 156, 6, 1149-1155.

2. Stark HJ, Szabowski A, Fusenig NE, Maas-Szabowski N (2004), Organotypic cocultures as skin equivalents: A complex and sophisticated in vitro system. Biol Proced Online 6, 55-60.

3. El Ghalbzouri A, Gibbs S, Lamme E, Van Blitterswijk C, Ponec M (2002), Effect of fibroblasts on epidermal regeneration. Br J Dermatol 1472, 230-243.

4. Sun T, Jackson S, Haycock JW, MacNeil S (2006), Culture of skin cells in $3 \mathrm{D}$ rather than $2 \mathrm{D}$ improves their ability to survive exposure to cytotoxic agents. J Biotechnol 122, 3, $372-381$.

5. Mertsching H, Weimer M, Kersen S, Brunner H (2008), Human skin equivalent as an alternative to animal testing. GMS Krankenhhyg Interdiszip 3, 1, Doc11. PMC2831516.

6. Ponec M (2002), Skin constructs for replacement of skin tissues for in vitro testing. Adv Drug Deliv Rev 54, 1, 19-30.

7. Ponec M, Boelsma E, Gibbs S, Mommaas M (2002), Characterization of reconstructed skin models. Skin Pharmacol Appl Skin Physiol 15, 1, 14-17.

8. Groeber F, Holeiter M, Hampel M, Hinderer S, SchenkeLayland K (2011), Skin tissue engineering - in vivo and in vitro applications. Adv Drug Deliv Rev 63, 4-5, $352-366$

9. Khorshid FA (2005), Comparative study of keloid formation in humans and laboratory animals. Med Sci Monit 11, 7, BR212-219.

10. European Commission, SCCS (Scientific Committee on Consumer Safety) (2016), SCCS notes of guidance for the testing of cosmetic ingredients and their safety evaluation 9th revision. SCCS 1564, 15, 1-151.

11. Becker RA, Borgert CJ, Webb S, Ansell J, Amundson S, Portier CJ, Goldberg A, Bruner LH, Rowan A, Curren RD (2006), Report of an ISRTP Workshop: Progress and barriers to incorporating alternative toxicological methods in the US. Regul Toxicol Pharmacol 46, 1, 18-22.

12. Cukierman E, Pankov R, Stevens DR, Yamada KM (2001), Taking cell-matrix adhesions to the third dimension. Science 294, 5547, 1708-1712.

13. Grinnell F (1976), Biochemical analysis of cell adhesion to a substratum and its possible relevance to cell metastasis. Prog Clin Biol Res 9, 227-236.

14. Bissell MJ, Hall HG, Parry G (1982), How does the extracellular matrix direct gene expression? J Theor Biol 99, 1, 31-68.

15. Yang J, Balakrishnan A, Hamamoto S, Beattie CW, Gupta TK, Wellings SR, Nandi S (1986), Different mitogenic and phenotypic responses of human breast epithelial cells grown in two versus three dimensions. Exp Cell Res 167, 2, $563-569$.

16. Lin CQ, Bissell MJ (1993), Multi-faceted regulation of cell differentiation by extracellular matrix. FASEB J 7, 9, 737-743.

17. Smalley KS, Lioni M, Herlyn M (2006), Life ins't flat: Taking cancer biology to the next dimension. In Vitro Cell Dev Biol Anim 42, 8-9, 242-247.

18. Grinnell F (2008), Fibroblast mechanics in three-dimensional collagen matrices. J Bodyw Mov Ther 12, 3, 191-193.

19. Horning JL, Sahoo SK, Vijayaraghavalu S, Dimitrijevic S, Vasir JK, Jain TK, Panda AK, Labhasetwar V (2008), 3-D tumor model for in vitro evaluation of anticancer drugs. Mol Pharm 5, 5, 849-862. 
20. Brücher BLDM, Jamall IS (2019), Synopsis: Special Issue on "Disruption of signaling homeostasis induced crosstalk in the carcinogenesis paradigm Epistemology of the origin of cancer". 4open 2, 28, 1-30.

21. Mazzoleni G, Di Lorenzo D, Steimberg N (2009), Modelling tissues in 3D: the next future of pharmaco-toxicology and food research? Genes Nutr 4, 1, 13-22.

22. Mathes SH, Ruffner H, Graf-Hausner U (2014), The use of skin models in drug development. Adv Drug Deliv Rev 69-70, 81-102.

23. Bilousova G, Chen J, Roop DR (2011), Differentiation of mouse induced pluripotent stem cells into a multipotent keratinocyte lineage. J Invest Dermatol 131, 4, 857-864.

24. Itoh M, Umegaki-Arao N, Guo Z, Liu L, Higgins CA, Christiano AM (2013), Generation of 3D Skin Equivalents Fully Reconstituted from Human Induced Pluripotent Stem Cells (iPSCs). PLoS One 8, 10, e77673. https://doi.org/ 10.1371/journal.pone.0077673.

25. Randall MJ, Jüngel A, Rimann M, Wuertz-Kozak K (2018), Advances in the biofabrication of $3 \mathrm{D}$ skin in vitro: Healthy and pathological models. Front Bioeng. Biotechnol 6, 154. https://doi.org/10.3389/journal.fbioe.2018.00154.

26. Brohlin M, Kelk P, Wiberg M, Kingham PJ (2017), Effects of a defined xeno-free medium on the growth and neurotrophic and angiogenic properties of human adult stem cells. Cytotherapy 19, 5, 629-639.

27. De Corte P, Verween G, Verbeken G, Rose T, Jennes S, De Coninck A, Roseeuw D, Vanderkelen A, Kets E, Haddow D (2012), Feeder layer- and animal product-free culture of neonatal foreskin keratinocytes: improved performance, usability, quality and safety. Cell Tissue Bank 131, 175-189.

28. Freshney RI (2016), Culture of animal cells: a manual of basic techniques and specialized applications, Wiley, New Jersey.

29. Boehnke K, Mirancea N, Pavesio A, Fusenig NE, Boukamp P, Stark HJ (2007), Effects of fibroblasts and microenvironment on epidermal regeneration and tissue function in long-term skin equivalents. Eur J Cell Biol 86, 11-12, 731-746.

30. Balasubramani M, Kumar TR, Babu M (2001), Skin substitutes: a review. Burns 27, 5, 534-544.

31. El Ghalbzouri A, Lamme E, Ponec M (2002), Crucial role of fibroblasts in regulating epidermal morphogenesis. Cell Tissue Res 310, 189-199.

32. El Ghalbzouri A, Jonkman MF, Dijkman R, Ponec M (2005), Basement membrane reconstruction in human skin equivalents is regulated by fibroblasts and/or exogenously activated keratinocytes. J Invest Dermatol 124, 1, 79-86.

33. Andriani F, Margulis A, Lin N, Griffey S, Garlick JA (2003), Analysis of microenvironmental factors contributing to basement membrane assembly and normalized epidermal phenotype. J Invest Dermatol 120, 6, 923-931.

34. Lee DY, Cho KH (2005), The effects of epidermal keratinocytes and dermal fibroblasts on the formation of cutaneous basement membrane in three-dimensional culture systems. Arch Dermatol Res 296, 7, 296-302.

35. Maas-Szabowski N, Shimotoyodome A, Fusenig NE (1999), Keratinocyte growth regulation in fibroblast cocultures via a double paracrine mechanism. J Cell Sci 112, 12, 1843-1853.

36. Maas-Szabowski N, Stark HJ, Fusenig NE (2000), Keratinocyte growth regulation in defined organotypic cultures through IL-1-induced keratinocyte growth factor expression in resting fibroblasts. J Invest Dermatol 114, 6, 1075-1084.

37. Sampias C, Rolls G (2019), H\&E Staining Overview: A Guide to Best Practices, Richmond, Leica Biosystems.

38. Nagashima K, Zheng J, Parmiter D, Patri AK (2011), Biological tissue and cell culture specimen preparation for TEM nanoparticle characterization. Methods Mol Biol 697, $83-91$.
39. Sharma S, Poddar R, Sen P, Andrews J (2008), Effect of vitamin $\mathrm{C}$ on collagen biosynthesis and degree of birefringence in polarization sensitive optical coherence tomography (PS-OCT). Afr J Biotechnol 7, 12, 2049-2054.

40. Chapman JA (1985), The banding pattern of collagen, in: R. Garrone (Ed.), Biology of Invertebrate and Lower Vertebrate Collagens Bairati A, Springer, Boston.

41. Duplan-Perrat F, Damour O, Montrocher C, Peyrol S, Grenier G, Jacob MP, Braye F (2000), Keratinocytes influence the maturation and organization of the elastin network in a skin equivalent. J Invest Dermatol 114, 2, 365-370.

42. Ehrlich HP, Krummel TM (1996), Regulation of wound healing from a connective tissue perspective. Wound Repair Regen 4, 2, 203-210.

43. Hur GY, Seo DK, Lee JW (2014), Contracture of skin graft in human burns: effect of artificial dermis. Burns 40, 8, 1497-1503.

44. Jackson SM, Williams ML, Feingold KR, Elias PM (1993), Pathobiology of the stratum corneum. West J Med 158, 3, 279-285.

45. Casale C, Imparato G, Urciuolo F, Netti PA (2016), Endogenous human skin equivalent promotes in vitro morphogenesis of follicle-like structures. Biomaterials 101, 86-95.

46. Segal N, Andriani F, Pfeiffer L, Kamath P, Lin N, Satyamurthy K, Egles C, Garlick JA (2008), The basement membrane microenvironment directs the normalization and survival of bioengineered human skin equivalents. Matrix Biol 27, 3, 163-170.

47. Idrees A, Chiono V, Ciardelli G, Shah S, Viebahn R, Zhang X, Salber J (2018), Validation of in vitro assays in three-dimensional human dermal constructs. Int $\mathrm{J}$ Artif Organs 41, 11, 779-788.

48. Bikle DD, Xie Z, Tu CL (2012), Calcium regulation of keratinocyte differentiation. Expert Rev Endocrinol Metab 7, 4, 461-472.

49. Menon GK, Grayson S, Elias PM (1985), Ionic calcium reservoirs in mammalian epidermis: ultrastructural localization by ion-capture cytochemistry. J Invest Dermatol 84, 6, 508-512.

50. Ponec M, Weerheim A, Kempenaar J, Mommaas AM, Nugteren DH (1988), Lipid composition of cultured human keratinocytes in relation to their differentiation. J Lipid Res 29, 7, 949-961.

51. Grubauer G, Elias PM, Feingold KR (1989), Transepidermal water loss: the signal for recovery of barrier structure and function. J Lipid Res 30, 3, 323-333.

52. Kopan R, Traska G, Fuchs E (1987), Retinoids as important regulators of terminal differentiation: examining keratin expression in individual epidermal cells at various stages of keratinization. J Cell Biol 105, 1, 427-440.

53. Kolli S, Lako M, Figueiredo F, Mudhar H, Ahmad S (2008), Loss of corneal epithelial stem cell properties in outgrowths from human limbal explants cultured on intact amniotic membrane. Regen Med 3, 3, 329-342.

54. Meller D, Pires R, Tseng S (2002), Ex vivo preservation and expansion of human limbal epithelial stem cells on amniotic membrane cultures. Br J Ophthalmol 86, 4, 463-471.

55. Pellegrini G, Traverso CE, Franzi AT, Zingirian M, Cancedda R, De Luca M (1997), Long-term restoration of damaged corneal surfaces with autologous cultivated corneal epithelium. Lancet 349, 9057, 990-993.

56. Tsai RJF, Li LM, Chen JK (2000), Reconstruction of damaged corneas by transplantation of autologous limbal epithelial cells. N Engl J Med 343, 2, 86-93.

57. Rheinwald JG, Green H (1975), Serial cultivation of strains of human epidemal keratinocytes: The formation of keratinizin colonies from single cells. Cell 6, 3, 331-343. 
58. Hayashi I, Larner J, Sato G (1978), Hormonal growth control of cells in culture. In vitro $14,1,23-30$.

59. Okada N, Kitano Y, Ichihara K (1982), Effects of cholera toxin on proliferation of cultured human keratinocytes in relation to intracellular cyclic AMP levels. J Invest Dermatol $79,1,42-47$.

60. Allen-Hoffmann BL, Rheinwald JG (1984), Polycyclic aromatic hydrocarbon mutagenesis of human epidermal keratinocytes in culture. Proc Natl Acad Sci USA 81, 24, 7802-7806.

61. Flaxman BA, Harper RA (1975), In vitro analysis of the control of keratinocyte proliferation in human epidermis by physiologic and pharmacologic agents. J Invest Dermatol 65, $1,52-59$.

62. Yu M, Bojic S, Figueiredo GS, Rooney P, De Havilland J, Dickinson A, Figueiredo FC, Lako M (2016), An important role for adenine, cholera toxin, hydrocortisone and triiodothyronine in the proliferation, self-renewal and differentiation of limbal stem cells in vitro. Exp Eye Res $152,113-122$.

63. Savini I, Rossi A, Duranti G, Avigliano L, Catani MV, Melino G (2002), Characterization of keratinocyte differentiation induced by ascorbic acid: Protein kinase $\mathrm{C}$ involvement and vitamin C homeostasis 1 . J Invest Dermatol 118, 2, 372-379.

64. Pullar J, Carr A, Vissers M (2017), The roles of vitamin C in skin health. Nutrients 9, 8, 866. https://doi.org/10.3390/ nu9080866.

65. Carlson MW, Alt-Holland A, Egles C, Garlick JA (2008), Three-dimensional tissue models of normal and diseased skin. Curr Protoc Cell Biol 41, 1, 19.9.1-19.9.17.

66. Egles C, Garlick JA, Shamis Y (2010), Three-dimensional human tissue models of wounded skin. Methods Mol Biol 585, $345-359$

67. Black AF, Bouez C, Perrier E, Schlotmann K, Chapuis F, Damour O (2005), Optimization and characterization of an engineered human skin equivalent. Tissue Eng 11, 5-6, 723-733.

68. Greenwald HS, Friedman EB, Osman I (2012), Superficial spreading and nodular melanoma are distinct biological entities: a challenge to the linear progression model. Melanoma Res 22, 1, 1-8.

69. Hill DS, Robinson ND, Caley MP, Chen M, O'toole EA, Armstrong JL, Przyborski S, Lovat PE (2015), A novel fullyhumanised 3D skin equivalent to model early melanoma invasion. Mol Cancer Ther 1411, 2665-2673.

70. Park JH, Park YJ, Kim SK, Kwon JE, Kang HY, Lee ES, Choi JH, Kim YC (2016), Histopathological differential diagnosis of psoriasis and seborrheic dermatitis of the scalp. Ann Dermatol 28, 4, 427-432.

71. Dimarco RL, Su J, Yan KS, Dewi R, Kuo CJ, Heilshorn SC (2014), Engineering of three-dimensional microenvironments to promote contractile behavior in primary intestinal organoids. Integr Biol (Camb) 6, 2, 127-142.

72. Sugihara H, Toda S, Miyabara S, Kusaba Y, Minami Y (1991), Reconstruction of the skin in three-dimensional collagen gel matrix culture. Vitro Cell Dev Biol 27A, 2, 142-146.

73. Santos M, Paramio JM, Bravo A, Ramirez A, Jorcano JL (2002), The expression of keratin k10 in the basal layer of the epidermis inhibits cell proliferation and prevents skin tumorigenesis. J Biol Chem 277, 21, 19122-19130.
74. Coulombe PA, Kopan R, Fuchs E (1989), Expression of keratin K14 in the epidermis and hair follicle: insights into complex programs of differentiation. J Cell Biol 109, 5, 2295-2312.

75. Fuchs E, Green H (1980), Changes in keratin gene expression during terminal differentiation of the keratinocyte. Cell 19,4, 1033-1042.

76. Al Saif F (2016), Dermatopathia pigmentosa reticularis: report of a new cases and literature review. Indian J Dermatol 61, 4, 468-475.

77. Thacher SM, Rice RH (1985), Keratinocyte-specific transglutaminase of cultured human epidermal cells: relation to cross-linked envelope formation and terminal differentiation. Cell 40, 3, 685-695.

78. Warhol M, Roth J, Lucocq JM, Pinkus G, Rice R (1985), Immuno-ultrastructural localization of involucrin in squamous epithelium and cultured keratinocytes. J Histochem Cytochem 33, 2, 141-149.

79. Mehrel T, Hohl D, Rothnagel JA, Longley MA, Bundman D, Cheng C, Lichti U, Bisher ME, Steven AC, Steinert PM, Yuspa SH, Roop DR (1990), Identification of a major keratinocyte cell envelope protein, loricrin. Cell 61, 6, 1103-1112.

80. Steven A, Bisher M, Roop D, Steinert P (1990), Biosynthetic pathways of filaggrin and loricrin - two major proteins expressed by terminally differentiated epidermal keratinocytes. J Struct Biol 104, 1-3, 150-162.

81. Nishiyama T, Amano S, Tsunenaga M, Kadoya K, Takeda A, Adachi E, Burgeson RE (2000), The importance of laminin 5 in the dermal - epidermal basement membrane. J Dermatol Sci 24, 1, S51-S59.

82. Elias PM, Menon GK, Grayson S, Brown BE (1988), Membrane structural alterations in murine stratum corneum: relationship to the localization of polar lipids and phospholipases. J Invest Dermatol 91, 1, 3-10.

83. Hohl D (1990), Cornified cell envelope. Dermatologica 180, 4 , 201-211.

84. Kee SH, Steinert PM (2001), Microtubule Disruption in Keratinocytes Induces Cell-Cell Adhesion through Activation of Endogenous E-Cadherin. Mol Biol Cell 12, 7, 1983-1993.

85. Pegu S, Bodani JP, Lemire EG, Holfeld KI (2017), A novel keratin 10 gene mutation causing epidermolytic hyperkeratosis (bullous congenital ichthyosiform erythroderma) in a term neonate. Case Rep Perinat Med 6, 1, 20160050. https://doi.org/10.1515/crpm-2016-0050.

86. Khani P, Ghazi F, Zekri A, Nasri F, Behrangi E, Aghdam AM, Mirzaei H (2018), Keratins and epidermolysis bullosa simplex. J Cell Physiol 234, 1, 289-297.

87. Maestrini E, Monaco AP, Mcgrath JA, Ishida-Yamamoto A, Camisa C, Hovnanian A, Weeks DE, Lathrop M, Uitto J, Christiano AM (1996), A molecular defect in loricrin, the major component of the cornified cell envelope, underlies Vohwinkel's syndrome. Nat Genet 13, 70-77.

88. Harrison CA, Gossiel F, Layton CM, Bullock AJ, Johnson T, Blumsohn A, MacNeil S (2006), Use of an in vitro model of tissue-engineered skin to investigate the mechanism of skin graft contraction. Tissue Eng 12, 11, 3119-3133.

89. Michel M, L'heureux N, Pouliot R, Xu W, Auger FA, Germain L (1999), Characterization of a new tissueengineered human skin equivalent with hair. In Vitro Cell Dev Biol Anim 35, 6, 318-326.

Cite this article as: Idrees A, Schmitz I, Zoso A, Gruhn D, Pacharra S, et al. 2021. Fundamental in vitro 3D human skin equivalent tool development for assessing biological safety and biocompatibility - towards alternative for animal experiments. 4open, 4, 1. 